\title{
The Role of Traditional Chinese Herbal Medicines in Cancer Therapy - from TCM Theory to Mechanistic Insights
}

Authors

Affiliation
W. L. Wendy Hsiao, Liang Liu

Center for Cancer \& Inflammation Research, School of Chinese Medicine, Hong Kong Baptist University, Hong Kong, China
Key words

- Chinese herbal medicines

- traditional Chinese medicine

- anti-cancer drugs

- mechanism of action of Chinese herbal medicines received May 3, 2010

revised July 1,2010

accepted July 5, 2010

Bibliography

DOI http://dx.doi.org/

10.1055/s-0030-1250186

Published online July 15, 2010

Planta Med 2010; 76:

1118-1131 @ Georg Thieme

Verlag KG Stuttgart · New York . ISSN 0032-0943

\section{Correspondence}

Prof. W. L. Wendy Hsiao

School of Chinese Medicine Hong Kong Baptist University

Kowloon Tong

Kowloon

Hong Kong

People's Republic of China

Phone: + 85234112959

Fax: + 85234112461

bowhsiao@hkbu.edu.hk

liuliang@hkbu.edu.hk

\section{Abstract}

$\nabla$

Traditional Chinese medicine-based herbal medicines have gained increasing acceptance worldwide in recent years and are being pursued by pharmaceutical companies as rich resources for drug discovery. For many years, traditional Chinese medicines (TCM) have been applied for the treatment of cancers in China and beyond. Herbal medicines are generally low in cost, plentiful, and show very little toxicity or side effects in clinical practice. However, despite the vast interest and ever-increasing demand, the absence of strong evidence-based research and the lack of standardization of the herbal products are the main obstacles toward the globalization of TCM. In recent years, TCM research has greatly accelerated with the advancement of analytical technologies and methodologies. This review of TCM specifically used in the treatment of cancer is divided into two parts. Part one provides an overview of the philosophy, approaches and progress in TCMbased cancer therapy. Part two summarizes the current understanding of how TCM-derived compounds function as anticancer drugs.

\section{Abbreviations}

$\nabla$

AFAP-1: actin filament-associated protein 1

AIF: apoptosis-inducing factor

BAD: $\quad$ Bcl-2-associated death promoter

BAK: $\quad$ BCL2-antagonist/killer

BAX: $\quad$ BCL2-associated X protein

BCL2: $\quad$ B cell leukemia/lymphoma 2

BCL-XL: B cell leukemia/lymphoma xL

BID: $\quad$ BH3 interacting domain protein

BIM: $\quad \mathrm{Bcl}-2$ interacting mediator

Casp3: $\quad$ caspase 3

Casp6: $\quad$ caspase 6

Casp7: $\quad$ caspase 7

Casp8: $\quad$ caspase 8
Casp10: caspase 10

cytoC: cytochrome C

ERK1/2: extracellular signal-regulated kinase $1 / 2$

FADD: $\quad$ FAS-associated death domain

FASL: $\quad$ FAS ligand

FOXO1: forkhead box 1

Gp130: glycoprotein 130

GRB2: growth factor receptor-bound protein 2

HER2: human epidermal growth factor receptor 2

IкB: IkappaB

IKK: IkappaB kinase

IL-6: interleukin-6

IL-6R: interleukin-6 receptor

JAK: Janus kinase

MCL-1: myeloid cell leukemia sequence 1

MDM2: $\quad$ murine double minute 2

MEK1/2: $\quad$ MAP kinase kinase 1/2

MMP: matrix metalloproteinase

Mule: $\quad$ Mcl-1 ubiquitin ligase E3

NF-kB: nuclear factor-kappaB

NIK: $\quad$ MEK kinase 14

p90RSK: p90 ribosomal S6 kinase

PARP: $\quad$ poly (ADP-ribose) polymerase

PI3K: $\quad$ phosphoinositide 3-kinase

PIP3: phosphatidylinositol 3,4,5-trisphosphate

PUMA: $\quad$ p53 upregulated modulator of apoptosis

SH2: $\quad$ Src-homology 2

SOS: $\quad$ son of sevenless

STAT3: signal transducer and activator of transcription 3

t-BID: truncated $\mathrm{BH} 3$ interacting domain protein

TIMP: $\quad$ tissue inhibitor of matrix metalloproteinase

TNF $\alpha$ R1: $\quad$ TNF-alpha R1

TNF $\alpha$ R2: $\quad$ TNF-alpha R2

Topo I: topoisomerase I 
Topo II: topoisomerase II

TRADD: TNFRSF1A-associated death domain

TRAF2: TNF receptor-associated factor 2

TRAIL: TNF-related apoptosis-inducing ligand
TRAILR: TRAIL receptor

VEGF: vascular endothelial growth factor

VEGFR: vascular endothelial growth factor receptor

XIAP: $\quad \mathrm{X}$-linked inhibitor of apoptosis protein

\section{Introduction}

\section{$\nabla$}

\section{TCM views of cancer and the approach}

\section{toward cancer treatment}

Cancer is one of the major causes of mortality in humans throughout the world. According to a report dealing with the incidence and mortality of cancers in the USA, a total of 1479350 new cancer cases and 562340 deaths from cancer were projected to occur in 2009 [1]. For cancer treatment by conventional medicine, surgery, chemotherapy and radiotherapy have been the primary approaches, but they are not always effective. As of today, cancer is still the most threatening and difficult to treat disease. Cancerous conditions are well-known in the traditional Chinese medical system. In the classics of TCM, "Huang Di Nei Jing Di" (黃帝內經) published more than 2000 years ago, there are descriptions of the pathogenesis, appearances and treatment principles of tumors (瘤), such as muscle, tendon and bone carcinomas; however, this term does not differentiate between malignant and nonmalignant tumors. It was not until the Sung Dynasty (ca. $1300 \mathrm{AD}$ ) that the first reference to cancer - the Chinese word Ai (癌) meaning malignant carcinoma - first appeared in the ancient medical book “Wei Ji Bao Shu” (衛濟寶書). According to the theories of TCM, cancer is caused by imbalances between endogenous physical conditions of the body and exogenous pathogenic factors. The internal condition of the body plays a dominant role in the onset of cancer. In other words, factors can induce cancer only when the body's own defense system fails. Those pathogenic factors, in Chinese medicine terms, include accumulated toxins, heat and blood stasis, and they attack when a person is in a weak physical condition, without the strength to resist. Furthermore, malfunction of the body-mind communication network may also trigger the development of cancer [2]. So, TCM doctors view cancer as a systemic disease associated with the state of the whole body (or disturbance of the signaling network, to use a modern term). "Systemic" in the TCM doctors' views, means "state of the whole body". "Cancer is the manifestation of a breakdown in the body's ability to handle pathogenic factors, not a local disease of cells or organs." Accordingly, the treatment philosophy and strategy of TCM emphasizes holistic modulation and improvement of the whole body rather than removing the tumor mass or killing the cancerous cells. This treatment strategy is particularly enforced for cancer patients at the late stages. In these stages, the focus of treatment is extending the life expectancy and improving the quality of life of the patient; in other words, the focus is on the patient not the tumor mass (帶瘤生存).

The other major principle of TCM is the emphasis on an individual therapy. For the same type of cancer in different persons, the diagnosis and treatment schemes could be very different. This is called the principle of "treatment based on symptom pattern differentiation (辨證論治)". In other words, TCM doctors make the diagnosis and prepare a treatment scheme based on the assessment of the pattern of symptoms manifest in each individual. When herbs are called for, most commonly, several are used together, and the whole herbs are used, not purified compounds. Thus, in the prescription, there will be multiple effective compo- nents delivering a comprehensive, integrated treatment of cancer through multiple targets and their associated pathways. This approach is in line with the view of TCM that cancer is a systemic disease that requires a holistic approach and medicines that can produce therapeutic actions through multiple targets. While this approach differs from that of conventional medicine, the effects of treatment still come down to biochemistry. If treatments are effective, then there must be underlying mechanisms that can be investigated and verified scientifically. Understanding these mechanisms can help us expand the efficacy of both Western and Chinese medicines in a logical, rational way.

\section{Evaluation of the Therapeutic Effects of}

\section{TCM Herbal Medicines in Cancer Treatment -}

\section{The Benefits and the Obstacles}

\section{$\nabla$}

To provide the scientific basis for the effectiveness of TCM against cancer, a number of clinical and laboratory studies have been done in the past decades. However, due to various factors - including inconsistency in treatment schemes, the limited sampling sizes, and lack of quality assurance of the herbal products - well-designed randomized controlled trials (RCT) to prove the effectiveness of TCM as adjuvant therapy for cancer are scarce. In general, most of the published clinical studies are at evidence level III; in other words, they were trials without rigorous randomization or they involved single group pre-post, cohort, time series, or matched case-control studies [3]. As a result, there are a number of contradictory reports regarding the therapeutic effectiveness of TCM on the treatment of cancer.

In TCM prescriptions, herbs are generally used in combination as 'formulas', in the belief that the combinations enhance their benefits and simultaneously reduce side effects. With proper diagnosis and understanding of the component herbs, practitioners can adjust or customize the formulas to suit individual cancer patients. Through synergistic interactions between different effective ingredients, the herbal preparation, according to the clinical experiences, can exert its effects in several ways: (i) they can protect the noncancerous cells and tissues in the body from the possible damage caused by chemo/radiotherapy; (ii) they can enhance the potency of chemo/radiotherapy; (iii) they can reduce inflammatory and infectious complications in the tissues surrounding the carcinoma; (iv) they can enhance immunity and body resistance; (v) they can improve general condition and quality of life; and (vi) they can prolong the life span of the patients in the late stages of cancer ( $\bullet$ Fig. 1). So, for an evaluation of the effect and benefit of TCM therapy for cancer patients, all of these above-mentioned aspects need to be considered. A typical example of the synergistic, complex function of the herbs has been given in the study of PHY906, a Chinese herbal preparation made from the herbs of Scutellaria baicalensis, Paeonia lactiflora, Ziziphus jujuba and Glycyrrhiza glabra. PHY906 is derived from a classic herbal formula used for treatment of diarrhea and inflammatory conditions. Based on the properties and functions of Chinese medicinal herbs, each formula has a principle herb and adjuvant herbs. Here, the $S$. baicalensis acts as a principle herb, 


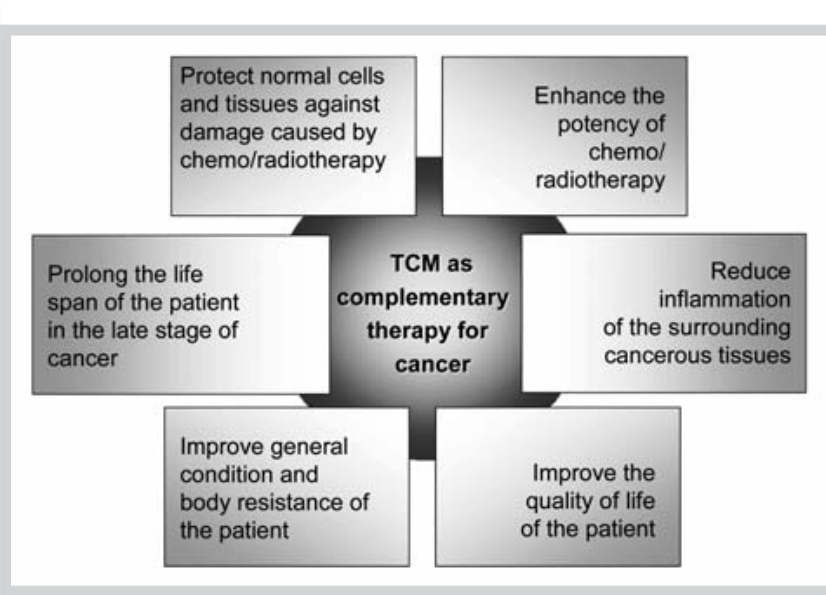

Fig. 1 Schematic presentation of the actions of TCM as adjuvant cancer therapy based on the theory and clinical practice of TCM.

while the rest of the herbs in the formula are assistant herbs. The phase I/IIA randomized clinical study of PHY906 demonstrated a reduction of gastrointestinal toxicity and enhancement of the tumoricidal effect of the chemotherapy in patients with advanced colorectal cancer [4]. Recently, it has been also found effective in increasing the therapeutic efficacy and reducing adverse effects of the cytotoxic drug capecitabine in patients with advanced hepatocellular carcinoma in a phase I/II clinical study [5]. Further animal study demonstrates that removing one or three herbs from the PHY906 formulation would dampen the effect of PHY906 in antitumor activity, reduces toxicity, and reduces the bioavailability of the principle herb (personal communication from Y.C. Cheng). Other than this, many studies have demonstrated that specific combinations of medicinal herbs can be synergistic with cytotoxic chemotherapy through both pharmacodynamic and pharmacokinetic interactions. In a cohort study, a combined treatment of traditional Chinese medicine and Western medicine (WM) or treatment with WM alone was conducted on 222 patients with stage II and III colorectal cancer after radical operation. The survival of WM alone and the WM and TCM combined treatment is 16 months and 26.5 months, respectively, in which WM was administrated as the routine protocol while a TCM formula was given according to the treatment principle of differentiation for symptom patterns [6].

In the past decade, there have been a few systematic reviews regarding the clinical trials against various cancers treated with a TCM formula. One systematic review on colorectal cancer showed that TCM therapy alone or in combination with chemotherapy is useful during the postoperation period in relieving intestinal obstruction, reducing postoperative ileus symptoms and urinary retention [7]. Another systematic review evaluating randomized clinical trails (RCT) of TCM oral therapy for hepatocellular cancers found that many RCTs, although the herbs they studied were effective, were not randomized [8]. With respect to non-small cell lung cancer (NSCLC), although it is one of the common cancers with the highest death rate, there are few reliable reports assessing TCM therapy. One RCT study done in China analyzing the survival time, Karnofsky score, clinical symptoms and adverse reactions shows that chemotherapy plus Kangliu Zengxiao Decoction (KLZXD) is able to prolong the survival time of patients up to 15.57 months versus 11.17 months in the patients treated with chemotherapy alone. The symptoms of fatigue and dyspnea and the adverse effects of leukopenia and dyspepsia caused by chemotherapy were reduced by treatment with KLZXD [9]. However, another study comparing the efficacy of TCM and chemotherapy against NSCLC found uncertain results. Nevertheless, TCM treatment seems to stabilize the tumor mass, relieve clinical symptoms, and elevate quality of life. It is also inexpensive and more convenient compared with the conventional chemotherapy [10]. With regard to advanced breast cancer, the herbal preparation Shenqi Fuzheng Injection (SFI), was demonstrated to alleviate bone marrow inhibition and cellular immunity suppression caused by chemotherapy, and to relieve clinical symptoms, raise the quality of life and prolong survival time compared to patients receiving only chemotherapy [11]. Similarly, a systematic review on nasopharyngeal carcinoma (NPC) suggests that TCM therapy is efficacious as a concomitant therapy for NPC patients, but rigorous controlled clinical trials are still required to confirm these results [12]. A report on TCM therapy for progressive gastric cancer shows some benefits to the patient but the study was not well designed, hence its conclusions are suspect [13]. Unfortunately, a systematic review on esophageal cancer shows no evidence to support the effectiveness of TCM therapy [14]. Based on the above reports, the efficacy of TCM therapy in cancers is still unproven by the current RCT standards.

Aside from the therapeutic effect of TCM therapy on suppressing cancer growth, other potential benefits to patients, such as enhancement of organ functions, improvement of quality of life, reduction of clinical symptoms including pain, and reduction of adverse effects of chemo-/radiotherapy are the indexes closely associated with the characteristics and advantages of TCM treatment as a supplementary remedy to the conventional chemo-/radiotherapy and those indexes have not always been taken into consideration in assessing the therapeutic outcome of TCM in RCT evaluations. For instance, a review analyzing the effectiveness of TCM for liver protection and chemotherapy completion among cancer patients shows that although there is no significant difference in outcome between the TCM-treated and the control groups, TCM therapy alongside the standard chemotherapy resulted in protection of liver function during the course of chemotherapy, as manifested by lower serum AST and ALT levels [15]. As we know, normal liver function is critical for successful completion of a standard course of chemotherapy. Thus, protection of liver function will not only protect the liver organ itself but will also enhance the cytotoxic potency of chemotherapeutic drugs. A systematic review on huangqi decoction in alleviation of chemotherapy side effects in colorectal cancer patients was recently reported [16]. Despite the low quality of the clinical studies, the results suggest that huangqi decoction may stimulate immunocompetent cells and decrease side effects in patients treated with chemotherapy. In addition to the alleviation of chemotherapy side effects, a reduction in cancer pain may also be a beneficial result from TCM treatment according to a recent systematic reviews of 115 articles on clinical research in TCM treatment for cancer pain management [17].

In recent years, improvement of quality of life and prolongation of survival time of cancer patients, whether the cancerous carcinoma disappears or not in the body, have become important factors for evaluating the benefits of any intervention for cancer patients. Another recent study evaluated the effect of the combined Ganji Recipe therapy with the Fructus Bruceae oil emulsion (FBE) intervention or the transhepatic arterial chemical embolization (TACE) on the quality of life and survival time of patients with advanced primary hepatic cancer. The results showed significant 
improvements in the quality of life and the life expectancy of the patients receiving Ganji and FBE treatment compared to patients treated with TACE or the Fructus Bruceae oil emulsion intervention alone [18]. Studies on non-small cell lung cancer indicate that TCM therapy is able to markedly reduce the adverse postradiation reactions and improve the quality of life of patients through intensive analysis using QLQ-C30, LC13, QLQ-C30 and LC30 questionnaires [19]. In another cohort study, the herbal preparation of Quxie Capsule was demonstrated to be effective in improving significantly the quality of life and in prolonging the survival of advanced colorectal cancer patients in an RCT study [1].

\section{Molecular Mechanisms of TCM-Based Herbal Medicines as Anticancer Drugs \\ $\nabla$}

Proponents of Chinese traditional medicines point out that these medicines, unlike Western drugs in which the therapeutic effects are derived from a single compound with a well-defined target, have synergistic pharmacological effects because each herbal formula consists of several herbs, each with specific therapeutic activities toward the disease or symptoms. Nonetheless, the majority of reports in the literature have taken a reductionist approach, namely working on mostly the TCM-derived pure compounds; few have studied extracts derived from a single herb, and very few have studied TCM formulations which are the form that have historically given the most benefit. At the time of this review, there were two comprehensive reviews published in 2003 on antitumor agents from TCM, focusing on both the chemical properties and the mechanisms of action of those compounds [20,21]. Therefore, this second part of the review will only touch upon a few studies in or before 2003, and will mainly cover reports after 2003 that appeared in the databases, including Ovid MEDLINE ${ }^{\circledR}$, AMED, CDSR, ACP Journal Club, DARE, CCTR, CLCMR, CLHTA, CLEED, EMBASE-DP, Global Health, restricted to TCM-based herbal medicines. Table 1 summarizes the articles selected out of $\sim 500$ original works on anticancer herbal medicines that meet the criteria mentioned above, i.e., (i) the article describes work on TCM-derived herbal medicine(s), and (ii) it is a mechanistic study at the molecular level. Due to the enormous volume of reports on apoptosis, this review in this area of study will mainly cover reports since 2006. In the review, we will also try to link the current understanding of anticancer TCM herbs based on the experimental and clinical studies with the understanding of the functions of the herbs based on TCM theory and concepts as developed during more than a thousand years of clinical experiences.

\section{Anticancer Effects and Underlying Mechanisms of TCM-Derived Compounds or Herbal Extracts \\ $\nabla$}

The anticancer herbal drugs can be divided into three categories based on their target: (i) drugs that uniquely target topoisomerases (Topos) and perturb DNA replication; (ii) drugs that kill tumor cells through apoptotic pathways; and (iii) drugs that alter signaling pathway(s) required for the maintenance of transforming phenotypes of the tumor cells. It is known that many natural products extracted from medicinal herbs show a direct killing effect on tumor cells. There is no exception for the compounds isolated from various traditional Chinese medicines claimed to have anticancer effects. Drugs targeting topoisomer- ase I \& II (Topo I \& II) as well as on pro-apoptotic pathways are known to induce cell death. Camptotheca acuminata (Xi Shu) is a TCM herb commonly used for cancer treatment in China. The compound camptothecin (CPT) isolated from the plant has been found to uniquely target Topo I, an enzyme which produces a DNA single-strand break in DNA replication. Interference of Topo I induces apoptosis and cell cycle perturbations [22-25]. This finding along with the discovery of natural product-derived taxol have been considered as historic achievements in natural products and have subsequently led to the identification of a series of TCM-derived compounds targeting Topo I \& II ( Table 1 ). Apoptosis is a common mode of action of chemotherapeutic agents, including the natural product-derived drugs [26,27]. It appears that this is true for TCM-derived compounds and their extracts. As shown in $\bigcirc$ Table 1, 85 out of 104 independent studies of herbal medicines derived from 62 different TCM-based herbal plants revealed that apoptosis is the key mode for cell killing in a wide variety of cancer cells upon the treatment with various tested herbal medicines. For example, solanine, a steroid alkaloid isolated from Solanum nigrum Linn. that is a TCM commonly used in treating digestive system cancer, was found to possess anticancer effects and induce apoptosis mediated by the inhibition of the expression of Bcl-2 pro-apoptotic protein [28]. The Antrodia camphorata crude extract (ACCE), an extract obtained from a rare traditional Chinese herbal medicine Zhan-Ku (a camphor tree mushroom), has shown rather significant inhibitory effects on the growth of various transitional cell carcinomas (TCC) including RT4, TSGH-8301, and T24 cell lines. Interestingly, at high concentrations, ACCE causes p53-independent overexpression of p21 with simultaneous down-alteration of pRb and senescence in RT4 cells. On the other hand, ACCE at a low concentration simultaneously downregulated Cdc2 and Cyclin B1 with suppression of the absolute migrating capability of the two cell lines TSGH-8301 and T24, and eventually caused cell death [29]. The concentration-dependent cellular responses seem to provide a new avenue to explore the anticancer effects of TCM, especially at nontoxic dosages. The dried root of Astragalus membranaceus (Huangqi) has a long history of medicinal use for immunodeficiency diseases and a relatively short history of equal effectivity for alleviating the adverse effects of chemotherapeutic drugs. Total Astragalus saponins induce growth inhibition and apoptosis in colon cancer cell line HT-29 and the xenograft [30]. Artemisinin is the active principle isolated from a well-known TCM herb, Artimisia annua L. an effective antimalaria drug. Later, studies showed that artemisinin and its derivatives have profound anticancer effects as assessed both in in vitro and in vivo models [31]. Artesunate, a derivative of artemisinin was found to inhibit angiogenesis and induce apoptosis through p53-dependent and independent pathways. The drug also inhibits NF-kappaB and downregulates the anti-apoptotic $\mathrm{Bcl}-2$ while it activates the pro-apoptotic Bax. Coptidis rhizoma (huanglian) and its major active component, berberine, were the most extensively studied herb of the last decade. Berberine as well as Huanglian show diverse biological activities, including antibacterial, anti-inflammatory, antiangiogenesis and pro-apoptotic activities [32]. A recent study elegantly demonstrated that berberine directly targets and modifies cysteine 179 of IkappaB kinase (IKK), leading to the downregulation of NFkappaB and its series of target genes in anti-apoptosis, cell proliferation, inflammation and invasion [33]. There are two major pathways by which chemotherapeutic agents can induce apoptosis of the treated cells. One is known as the mitochondria-mediated pathway, which is activated by cyto- 
Table 1 Examples of anticancer compounds or extracts derived from traditional Chinese herbal medicines with known molecular mechanism of action.

\begin{tabular}{|c|c|c|c|c|c|}
\hline Affected molecules and pathways & $\begin{array}{l}\text { In vitro and/or } \\
\text { in vivo system }\end{array}$ & Main TCM herbs & Active component ${ }^{*}$ & Type of compound & Ref. \\
\hline \multicolumn{6}{|l|}{ Topoisomerase I \& II inhibitors } \\
\hline$\downarrow \downarrow$ Topol & cancer cells & Camptotheca acuminata ${ }^{5}$ & camptothecins & alkoloid & {$[22,52]$} \\
\hline$\downarrow \downarrow$ Topo II & CHO cells & Chan'su' & bufalin & cardiac steroid & [53] \\
\hline - $\downarrow$ Topo I \& II \& other targets & & & & low molecular weight & [20] \\
\hline$\downarrow \downarrow$ Topo II & & Poria $\operatorname{cocos}^{3}$ & dehydroebriconic acid & triterpenoid & [54] \\
\hline - $\downarrow$ Topo I \& II; $\uparrow$ ERK, JNK; $\rightarrow$ apoptosis & hepatoma cells & Ganoderma ${ }^{3}$ & genodermic acid & triterpenoid & [55] \\
\hline$\downarrow \downarrow$ Topo I & in vitro assays & Daphne genkwa ${ }^{5}$ & yuanhuacines & diterpenoid & [56] \\
\hline$\checkmark \downarrow$ Topo II, ROS & cells, \& xenografts & & salvicine & diterpenoid & [57] \\
\hline $\begin{array}{l}\text { HER2, TNFR, Fas; } \downarrow \text { DNA synthesis; } \\
\rightarrow \text { apoptosis }\end{array}$ & lung cancer cell lines & Solanum ${ }^{1}$ & & alkaloid & {$[58]$} \\
\hline $\begin{aligned} & \text { Antioxidant; } \downarrow \text { NF-kappaB, Cox-2, } \\
& \text { PLase C \& Ca }{ }^{++} \text {signaling }\end{aligned}$ & various cancer cell lines & Scutellaria ${ }^{1}$ & $\begin{array}{l}\text { wogonin, baicalein, } \\
\text { baicalin }\end{array}$ & flavones & [59] \\
\hline 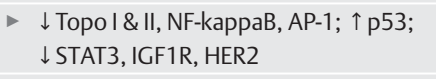 & & Many & luteolin & flavonoids & {$[60]$} \\
\hline \multicolumn{6}{|l|}{ Apoptosis } \\
\hline 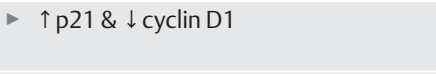 & $\begin{array}{l}\text { breast cancer cells } \\
\text { MDA-MB-231 }\end{array}$ & Coriolus versicolor ${ }^{3}$ & $\begin{array}{l}\text { polysaccharides pep- } \\
\text { tide }\end{array}$ & polysaccharides & {$[61]$} \\
\hline - Microarrays-detected genes & H lung cancer $A 549$ cells & Scutellaria barbata ${ }^{1}$ & crude extract & naphthoquinone & {$[62]$} \\
\hline - $\downarrow$ Telomerase \& Bcl-2, $\uparrow$ Bax & leukemia K562 & Rabdosia rebescens ${ }^{1}$ & oridonin & diterpene & [63] \\
\hline$\downarrow \downarrow \mathrm{BCl}-2$ & Нер3В & Scutellaria barbata ${ }^{1}$ & pheophrbide & & [64] \\
\hline - $\downarrow \mathrm{Bcl}-2, \uparrow \mathrm{p} 53$ \& PARP-1 & MCF-7 & Curcuma xanthorrhiza ${ }^{2}$ & xanthorrhizol & sesquiterpenoid & {$[65]$} \\
\hline$\downarrow \downarrow \mathrm{BCl}-2 / \mathrm{Bcl}-\mathrm{XL}$ & MCF-7 & Patrinia scabiosaefolia ${ }^{1}$ & not known & crude extract & [66] \\
\hline - $\uparrow$ Caspases \& Bax & prostate PC-3 & Alismatis rhizoma ${ }^{4}$ & alisol B acetate & triterpene & [67] \\
\hline - $\downarrow \mathrm{BCl}-2, \uparrow$ Caspases \& Bax & lung cancer cells & Rabdosia rebescens ${ }^{1}$ & ponicidin & diterpenoid & [68] \\
\hline - $\uparrow$ PARP, caspase-3 & leukemia K562 & Sophora flarescens Ait. ${ }^{1}$ & matrine & alkaloid & [69] \\
\hline - $\uparrow$ Caspase-8, with trail & $\begin{array}{l}\text { cholangiocarcinoma } \\
\text { TRAlL-resistant cells }\end{array}$ & Tripterygium wilfordii ${ }^{5}$ & triepolide & diterpene & [70] \\
\hline - Cdk inhibitor & chronic myelocytic leukemia & Danggui longhui Wan\# & indirubin & alkaloid & [71] \\
\hline - $\downarrow$ JAK \& STAT phosphorylation & HTLV-1-T leukemia cells & Curcuma longa ${ }^{2}$ & curcumin & diarylheptanoid & [72] \\
\hline - $\uparrow$ Caspase-8, $\downarrow$ Bcl-XL & Hep G2, Нер 3B & Justicia procumbens ${ }^{7}$ & justicidin & & [73] \\
\hline - $\uparrow$ Caspase-3, $\downarrow$ MMP & $\mathrm{HL}-60$ & Curcuma longa ${ }^{2}$ & curcumin & diarylheptanoid & [74] \\
\hline - $\uparrow$ p53 and Bax, $\downarrow$ Bcl-2 & lung cancer cells & Ganoderma lucidum ${ }^{3}$ & ganoderic acid T & triterpenoid & [75] \\
\hline - $\uparrow$ Caspase-3 \& -8 , cleavage of PARP & $\begin{array}{l}\text { pancreatic carcinoma } \\
\text { PANC-1 \& HeLa cells }\end{array}$ & Tripterygium wilfordii ${ }^{5}$ & triptolide & diterpene & [76] \\
\hline - $\uparrow$ Caspase-3 \& -9, PARP-1 & Jurkat T cells & Albizia julibrissin ${ }^{6}$ & crude extract & NA & [77] \\
\hline - $\uparrow$ Caspase-2 \& -3 & $\mathrm{HL}-60$ & Oldenlandia diffusa ${ }^{1}$ & crude extract & NA & [78] \\
\hline - $\uparrow$ Caspase-3 \& PARP & MCF-7 & Antrodia camphorata ${ }^{3}$ & crude extract & NA & [79] \\
\hline - $\uparrow \mathrm{p} 53, \downarrow \mathrm{Bcl}-2, \uparrow \mathrm{Bax}, \downarrow$ NF-kappaB & in vitro \& in vivo & Artemisia annua $L^{1}$ & artemisinin & sesquiterpene & [31] \\
\hline - $\downarrow$ PI3K/AKT, $\downarrow$ FOXO \& GSK3 pathways & osteosarcoma cells & Albatrellus confluens $^{7}$ & grifolin & & [80] \\
\hline - $\uparrow \mathrm{ROS}, \downarrow \mathrm{p}-\mathrm{Rb}, \downarrow \mathrm{p} 27$ & H HepG2 & Zizyphus jujuba fruit ${ }^{3}$ & organic crude extract & & [81] \\
\hline - $\uparrow$ Caspase-3 \& PARP & colon cancer cells HT-29 & & pseudolaric acid B & diterpenoid & [82] \\
\hline - $\uparrow$ Fas-mediated pathway & MCF-7 & Pterocarya stenoptera ${ }^{7}$ & pterocamin A & & [83] \\
\hline - $\uparrow$ Caspases & HeLa, A549, HepG2 \& SW480 & Cremanthodium humile 7 & crude extract & NA & [84] \\
\hline - $\uparrow$ Caspase-3, $-9,-4$. BIP \& CHOP (ER) & HL-60 & & trichosanthin & & [85] \\
\hline - $\uparrow$ Caspase-3 \& -7 & thyroid carcinoma cells & Stemona tuberosa Lour ${ }^{4}$ & organic fraction & NA & [86] \\
\hline - $\uparrow$ Bax, $\downarrow$ Bcl-2, p53-independent & glioma cells & Tripterygium wilfordii ${ }^{5}$ & triptolide & diterpene & [87] \\
\hline 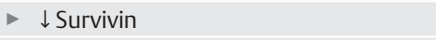 & lung cancer cells & Artemisia annua $^{1}$ & dihydroartemisinin & sesquiterpene & [88] \\
\hline - $\uparrow$ Caspase-3 \& -9 & neuroblastoma & Angelica keiskei ${ }^{7}$ & isobavachalcone & chalcones & [89] \\
\hline not clear & HL-60 & Oroxylum indicum ${ }^{1}$ & baiclein & flavonoid & [90] \\
\hline - $\uparrow$ Bax, $\downarrow$ Bcl-2, caspase-8 & HL-60 & Garcinia hanburyi ${ }^{5}$ & gambogic acid derivative & & [91] \\
\hline - $\uparrow$ PARP cleavage & HepG2 & Schisandra propinqua ${ }^{7}$ & schisandrolic acid & triterpenoid & [92] \\
\hline - $\uparrow$ Caspase-3, cleavage of PARP & HT-29 colon cancer cells & Astragalus membranaceus $^{1}$ & Astragalus saponins & saponins & [30] \\
\hline$\rightarrow$ Differentiation, GFAP, $\downarrow$ nestin & glioma cells & Danshen $^{2}$ & tanshinone IIA & phenanthraquinone & [93] \\
\hline - Caspase-3, PARP\& $\mathrm{p38}, \downarrow$ MAPK & HepG2 & 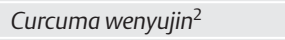 & furanodiene & & [94] \\
\hline - $\uparrow$ Capspase-like activities & Всар37 & Trichosanthes kirilowlii ${ }^{5}$ & $\begin{array}{l}\text { 23,24-dihydrocucur- } \\
\text { bitancin B }\end{array}$ & & [95] \\
\hline$\downarrow \downarrow$ Glutathione, enhance $\mathrm{As}_{2} \mathrm{O}_{3}$ toxicity & leukemia cells, HL-60 & Isodon melissoides 7 & melissoidesin G & diterpenoid & [96] \\
\hline - $\uparrow \mathrm{p} 38, \uparrow$ capases- $-9,-8,-3,-2$ & leukemia cells & Aglaia $^{7}$ & rocaglamide & rocaglamide & [97] \\
\hline - $\uparrow \mathrm{PLC}, \mathrm{Ca}^{++}$overload & $\begin{array}{l}\text { T-lymphocytes, in vitro \& } \\
\text { xenograft }\end{array}$ & Scutellaria baicalensis ${ }^{1}$ & wogonin & flavenoid & [37] \\
\hline - Synergize with TRAIL via $\downarrow$ XIAP \& DR5 & AML & & triptolide & diterpene & [98] \\
\hline - via DNA damage \& AIF pathway & HCT-116 & $\begin{array}{l}\text { Ephemerantha } \\
\text { lonchophylla }\end{array}$ & denbinobin & phenanthraquinone & [99] \\
\hline
\end{tabular}


Table 1 Examples of anticancer compounds or extracts derived from traditional Chinese herbal medicines with known molecular mechanism of action. continued

\begin{tabular}{|c|c|c|c|c|c|}
\hline Affected molecules and pathways & $\begin{array}{l}\text { In vitro and/or } \\
\text { in vivo system }\end{array}$ & Main TCM herbs & Active component ${ }^{*}$ & Type of compound & Ref. \\
\hline $\begin{array}{l}\text { T Bax/Bcl-2 ratio, caspase- }-9,-3, \& \\
\text { PARP-1 }\end{array}$ & glioblastoma cells & & berberine & isoquinoline alkaloid & {$[100]$} \\
\hline - $\uparrow$ Bax, Bak, \& Bad; $\downarrow$ Bcl-xL \& Mcl-1 & prostate $\mathrm{PC}-3$ xenograft & Magnolia officinalis ${ }^{4}$ & honokiol & lignan & {$[101]$} \\
\hline • $\downarrow$ ERK \& MAPK phosphorylation & PC3 prostate cancer cells & Antrodia camphorata ${ }^{3}$ & crude extract & NA & {$[102]$} \\
\hline$\triangleright \downarrow \mathrm{BCl}-2$ & HepG2 & Solanum nigrum Linn. ${ }^{1}$ & solanine & steroid alkaloid & [28] \\
\hline - $\uparrow$ Caspase-3; $\downarrow$ AKT pathway & breast cancer cells & Cordyceps militaris ${ }^{7}$ & crude extract & NA & {$[103]$} \\
\hline $\begin{aligned}= & \uparrow p 53 \& \mathrm{p} 21 ; \uparrow \text { caspase- } 3 ; \downarrow \text { Bcl-2, } \\
& \text { p-Rb, cdk 2/4 \& E2F }\end{aligned}$ & uterine leiomyoma cells & & isoliquiritigenin & flavonoid & [104] \\
\hline $\begin{aligned} & \uparrow \text { Caspase-8, -9,-7 \& -3; } \uparrow \text { PARP; } \\
& \downarrow \text { BCl-xL, }\end{aligned}$ & $\begin{array}{l}\text { prostate cancer cells } \\
\text { DU145 }\end{array}$ & Saussurea lappa ${ }^{6}$ & dehydrocostus lactone & & [105] \\
\hline - $\uparrow$ Caspase-3, AIF \& Bad; $\downarrow$ AKT & $\begin{array}{l}\text { lung adenocarcinoma } \\
\text { A549 }\end{array}$ & $\begin{array}{l}\text { Ephemerantha } \\
\text { lonchophylla }\end{array}$ & denbinobin & & {$[106]$} \\
\hline > $\uparrow$ Caspase-3 & pancreatic PANC-1 & Brucea javanica ${ }^{1}$ & Fructus Bruceae & & [107] \\
\hline - $\uparrow$ p53 \& p21; caspase-3 \& PARP & gastric carcinoma AGS cells & Strychni semen ${ }^{5}$ & water extract & & [108] \\
\hline - $\uparrow$ Caspase-3 \&-9; PARP & HL-60 cells & Schizandrae chinensis ${ }^{3}$ & $\begin{array}{l}\text { deoxy- \& gamma- } \\
\text { schizandrin }\end{array}$ & lignan & [109] \\
\hline - $\uparrow p 21$, caspase-3 \& -9 & prostate LNCaP cancer cells & Stellera chamaejasme L. ${ }^{5}$ & neochamaejasmin A & flavonoid & {$[110]$} \\
\hline $\begin{array}{l}\text { - } \uparrow \text { Caspases-8, }-9,-3 ; \downarrow \text { Bcl-2 \& } \\
\text { NF-kappaB }\end{array}$ & $\begin{array}{l}\text { oral squamous carcinoma } \\
\text { cells }\end{array}$ & & shikonin & naphthoquinone & [111] \\
\hline$>\uparrow \mathrm{Ca}^{++} \& \mathrm{p} 38$ & lung cancer PC-14 cells & Artemisia annua ${ }^{1}$ & dihydroartemisinin & sesquiterpene & {$[112]$} \\
\hline $\begin{array}{l}\quad \downarrow \text { NF-kappaB pathway via } \downarrow \text { IKB } \alpha \\
\text { kinase (IKK) }\end{array}$ & jurkat leukemic cells & & berberine & $\begin{array}{l}\text { isoquinoline alka- } \\
\text { loid }\end{array}$ & [33] \\
\hline $\begin{array}{l}\downarrow \text { JAK/STAT pathway; } \\
\uparrow \text { caspase- } 3,-8 \&-9\end{array}$ & $\begin{array}{l}\text { U266 multiple myeloma } \\
\text { cells }\end{array}$ & $\begin{array}{l}\text { Mylabria phalerata Pallas } \\
\text { (insect) }\end{array}$ & cantharidin & monoterpene & {$[113]$} \\
\hline - $\uparrow$ Bax; $\downarrow$ BCl-2; stimulate IL-2 \& TNF- $\alpha$ & $\begin{array}{l}\text { hepatocellular carcinoma } \\
\text { HepA cells }\end{array}$ & Paeonia moutan ${ }^{1}$ & paeonol & $\begin{array}{l}\text { phenolic com- } \\
\text { pound }\end{array}$ & [38] \\
\hline - $\downarrow$ IKK kinase activity; $\downarrow$ NFkappaB & carcinoma $\mathrm{KB}$ cells & Euphorbia fischeriana ${ }^{5}$ & 17-acetoxyjolkinolide B & & {$[114]$} \\
\hline - $\uparrow$ Caspase-3 \& -9, PARP; $\downarrow$ Bcl-2; $\uparrow$ Bax & multiple myeloma cells & Alpinia pricei Hayata ${ }^{7}$ & crude extract & NA & {$[115]$} \\
\hline $\begin{array}{l}\downarrow \quad \downarrow \text { PI3k/AKT \& NF-kappaB; enhance } \\
\text { Dex-effectiveness }\end{array}$ & HeLa & Tripterygium wilfordii ${ }^{5}$ & triptolide & diterpenoid & [116] \\
\hline > Caspase-3 & HCT116 colon cancer cells & Zingiber zerumbet ${ }^{7}$ & zerumbone & & [117] \\
\hline $\begin{array}{l}\text { Enhance 5-FU-induced caspase-6 \& } \\
\text { apoptosis }\end{array}$ & $\begin{array}{l}\text { gastric carcinoma } \\
\text { SCG-7901cells }\end{array}$ & Erigeron breviscapus ${ }^{1}$ & scutellarin & flavonoid & [118] \\
\hline - $\uparrow$ Fas/FasL; caspase-3 & oral squamous cell carcinoma & Sophora flavescens Ait ${ }^{1}$ & matrine & alkaloid & [119] \\
\hline - $\uparrow$ Caspases, PARP; $\uparrow$ ROS & tongue cancer SCC-4 cells & $\begin{array}{l}\text { Anisomeles indica (L) } \\
\text { Kuntze }^{7}\end{array}$ & ovatodiolide & diterpenoid & {$[120]$} \\
\hline - $\uparrow \mathrm{Bax} / \mathrm{Bcl}-2$ ratio; $\uparrow \mathrm{ROS}$ & colon cancer HCT116 cells & rhubarb ${ }^{2}$ & rhein & anthraquinone & [121] \\
\hline $\begin{array}{l}\text { Enhance trail-cytotoxicity through } \uparrow \\
\text { p53 \& puma mediated by ROS }\end{array}$ & $\begin{array}{l}\text { breast MDA-MB-453 cancer } \\
\text { cells }\end{array}$ & & wogonin & flavonoid & {$[122]$} \\
\hline $\begin{array}{l}\downarrow \text { ErbB- } 2 \text { pathway; } \uparrow \text { caspase-3 \& } \\
\text { PARP; } \uparrow \text { proapoptotic Bim }\end{array}$ & $\begin{array}{l}\text { MDA-MB- } 453 \text { breast cancer } \\
\text { cells }\end{array}$ & Cleistocalyx operculatus ${ }^{7}$ & on-III & & [123] \\
\hline - $\uparrow$ MAG-related mitochondrial pathway & HepG2 liver cancer cells & Scutellaria baicalensis ${ }^{1}$ & oroxylin A & flavonoid & {$[124]$} \\
\hline D Caspase-3 & $\begin{array}{l}\text { lung adenocarcinoma } \\
\text { ASTC cells }\end{array}$ & Artemisia annua ${ }^{1}$ & dihydroartemisinin & sesquiterpene & [125] \\
\hline - $\uparrow \mathrm{Bax} / \mathrm{Bcl}-2$ ratio & $\begin{array}{l}\text { prostate cancer PC-3 \& } \\
\text { DU-145 cells \& xenograft } \\
\text { mouse model }\end{array}$ & Lycium barbarum 3 & $\begin{array}{l}\text { polysaccharide } \\
\text { fraction }\end{array}$ & polysaccharides & {$[126]$} \\
\hline - $\downarrow$ Bcl-2; caspase-3; $\uparrow$ PARP cleavage & lung carcinoma A549 cells & Panax notoginseng ${ }^{2}$ & water extract & NA & [127] \\
\hline - $\downarrow$ TNF $\alpha$ \& NF-kappaB; $\uparrow$ ROS, caspases & leukemia U937 cells & Dendrobium moniliforme $e^{3}$ & denbinobin & phenanthraquinone & {$[128]$} \\
\hline $\begin{array}{l}\text { † p21 \& p27; } \uparrow \text { caspase-3 \& PARP } \\
\text { cleavage }\end{array}$ & leukemia K562 cells & Vitex rotundifolia ${ }^{1}$ & casticin & & [129] \\
\hline - $\uparrow$ ROS, Egr-1 \& caspase-3 & human leukemia U937 cells & Platycodon grandiflorum 4 & platycodon $\mathrm{D}$ & triterpenoid & {$[130]$} \\
\hline - $\uparrow \mathrm{ROS}, \downarrow \mathrm{MMP}, \downarrow \mathrm{Bax} / \mathrm{Bcl}-2$ ratio & HT-29 colon cancer cells & Houttuynia cordata $^{1}$ & crude extract & NA & [131] \\
\hline • $\downarrow$ Rho GTPases, $\downarrow$ metastasis & $\begin{array}{l}\text { nasopharyngeal carcino- } \\
\text { ma, HONE1 }\end{array}$ & Coptidis rhizoma & berberine & alkaloid & {$[132]$} \\
\hline > $\downarrow$ IL-6 \& JAK/STAT3 & prostate LNCap cells & Euphorbia fischeriana ${ }^{5}$ & 17-hydroxyjolkinolide B & diterpenoid & [133] \\
\hline > $\uparrow$ Bax, $\uparrow p 53$, AKT \& JNK & $\begin{array}{l}\text { TRAMP prostate mouse } \\
\text { model \& LNCaP cells }\end{array}$ & Scutellaria barbata ${ }^{1}$ & crude extract & NA & [134] \\
\hline $\begin{aligned} & \uparrow \text { Caspase-3, -9; } \uparrow \text { PARP cleavage; } \\
& \downarrow \text { Bcl-2 }\end{aligned}$ & $\begin{array}{l}\text { ovarian cancer cell iline } \\
\text { SKOV3 \& in vivo SKOV3 } \\
\text { xenograft in mice }\end{array}$ & Rhizoma Paridis $^{1}$ & paris saponin 1 & saponin & {$[135]$} \\
\hline - $\uparrow$ Caspase-8, -9; $\uparrow$ Bax; $\downarrow$ Bcl-2 & HL-60 cells & Garcinia hanburyi ${ }^{5}$ & gambogic acid & & [136] \\
\hline - $\uparrow$ Caspase-8, -9; $\uparrow$ Bax; $\downarrow$ Bcl-2 & melanoma A375 cells & Garcinia hanburyi ${ }^{5}$ & gambogic acid & & [137] \\
\hline
\end{tabular}


Table 1 Examples of anticancer compounds or extracts derived from traditional Chinese herbal medicines with known molecular mechanism of action. continued

\begin{tabular}{|c|c|c|c|c|c|}
\hline Affected molecules and pathways & $\begin{array}{l}\text { In vitro and/or } \\
\text { in vivo system }\end{array}$ & Main TCM herbs & Active component ${ }^{*}$ & Type of compound & Ref. \\
\hline - $\downarrow \mathrm{BCl}-2$; Fas ligand and receptor & lung carcinoma A549 cells & Venenum bufonis (Chansu) $)^{5}$ & crude extract & NA & [138] \\
\hline$\downarrow \downarrow \mathrm{Bcl}-2 /$ Bax ratio; $\uparrow$ caspases & $\begin{array}{l}\text { macrophage RAW } 264.7 \& \\
\text { THP-1 cells }\end{array}$ & Vitex negundo $^{1}$ & vitexins & lignans & [139] \\
\hline \multicolumn{6}{|l|}{ Others } \\
\hline - $\downarrow$ PKA \& PKC, $\downarrow$ cdc2\&CKII & leukimia cells & Chan'su $^{5}$ & bufalin & cardiac steroid & [140] \\
\hline $\begin{array}{l}\downarrow \downarrow \text { PKC, } \downarrow \text { NFkappaB-related transcrip- } \\
\text { tion from microarray data }\end{array}$ & $\begin{array}{l}\text { breast cancer cells \& } \\
\text { xenograft }\end{array}$ & Coix lachryma jobi L. ${ }^{3}$ & not known & crude emusion & [141] \\
\hline $\begin{array}{l}\text { Induce senescence; } \uparrow \text { p21 \& } \downarrow \text { p-Rb; } \\
\\
\downarrow \text { cdc2 \& cyclin B1, cell death }\end{array}$ & $\begin{array}{l}\text { RT4, TSGH-8302 \& } \\
\text { T24 carcinoma }\end{array}$ & $\begin{array}{l}\text { Antrodia camphorata } \\
\text { (fungi) })^{3}\end{array}$ & crude extract & & [29] \\
\hline - Antiangiognesis; $\downarrow$ VEGF, VEGFR, MVC & & & running II & & [142] \\
\hline $\begin{array}{l}\text { Stronger than tamoxifen in ER+ \& } \\
\text { ER-cells }\end{array}$ & MCF-10A \& -7 breast cells & Salvia miltiorrhiza ${ }^{2}$ & tanshinone IIA & phenanthraquinone & [143] \\
\hline $\begin{array}{l}\text { Cytotoxicity through non-apoptosis } \\
\text { mean }\end{array}$ & $\begin{array}{l}\text { ER + MDA-MB-231 \& MCF-7 } \\
\text { cells }\end{array}$ & ${\text { Leonurus japonicus }{ }^{2}}^{2}$ & organic extract & NA & [144] \\
\hline $\begin{array}{l}\text { Induce autophagy } \& \text { autophage-re- } \\
\text { lated cell death; } \downarrow \text { Bcl-2 and mTOR }\end{array}$ & $\begin{array}{l}\text { LNCaP prostate cancer } \\
\text { cells }\end{array}$ & Glycyrrhiza glabra ${ }^{3}$ & $\begin{array}{l}\text { licorice \& licochal- } \\
\text { cone-A }\end{array}$ & & [145] \\
\hline
\end{tabular}

NA: not applied; $\uparrow$ : activation; $\downarrow$ : inhibition; $\rightarrow$ : induction; * Chemical structures of the active components are shown in $\bullet$ Fig. 3 ; ${ }^{\#}$ Aloe vera (15 g), Angelica sinensis (30 g), Coptis chinensis (30 g), Gardenia jasminoides (30 g), Gentiana scabra Bge. (15 g), Moschus berezovskii (1.5 g), Phellodendron amurense (30 g), Rheum officinale Baill (15 g), Saussurea lappa $(4.5 \mathrm{~g})$, Scutellaria baicalensis ( $30 \mathrm{~g}$ ); Classification of medicinal herbs based on the properties and actions of Chinese medicinal herbs (see $\bullet$ Table 2 for details): ${ }^{1}$ Medicine for heat clearance and detoxification; ${ }^{2}$ Medicinal for promoting blood circulation and eliminating stasis; ${ }^{3}$ Medicinal for strengthening qi; ${ }^{4}$ Medicinal for resolving phlegm and removing stasis; ${ }^{5}$ Medicinal with cytotoxic function; ${ }^{6}$ Medicinal for dispersing edema and relieving pain; ${ }^{7}$ Others

chrome c, followed by the release of cytochrome c and Apaf-1, and activation of caspases- 9 and -3 . The caspase- 3 then induces degradation of many signaling molecules, including a DNA repair molecule and poly-ADP-ribose polymerase (PARP), and leads to irreversible cell death. The second pathway, the extrinsic receptor-mediated pathway involves death receptors, such as FAS, TNF $\alpha$ R1 \& R2, and TrailR. The latter pathway plays a major role in immune responses, but a lesser role in the response to genotoxic stress. As shown in $\bigcirc$ Table 1, there are only a small number of TCM-derived compounds that cause apoptosis via the extrinsic pathway. For example, matrine, an alkaloid purified from the Chinese herb Sophora flavescens Ait that is known as an anti-inflammation, antifibrotic and anticancer drug from TCM, activates caspase-3 through Fas/FasL in a gastric cancer cell line [34]. Triptolide is a purified component isolated from Tripterygium wilfordii that has been effective in treating a variety of inflammatory and autoimmune diseases. Tripolide also shows potent antitumor properties [35]. A study has demonstrated that triptolide not only inhibits XIAP, a potent cellular caspase inhibitor elevated in acute myeloid leukemia (AML) and a factor causing resistance to TNF $\alpha$-related apoptosis-inducing ligand (TRAIL), but also activates p53 signaling and promotes apoptosis of AMLs. In a separate study [36], tripolide was also found to overcome desmethasone-resistance and to enhance bortezomib/PS-341-induced apoptosis. It is believed that tripolide acts through multiple signaling pathways, including the PI3K/Akt/NF-kappaB observed in human myeloma cells. Wogonin isolated from Huang-Qin (Scutellaria baicalensia Georgl.) showed phospholipaseC $\gamma 1$ - and $\mathrm{Ca}^{++}$-dependent apoptosis [37]. Paeonol from the root bark of the TCM herb Peoinia moutan is a TCM herb used to activate blood flow and remove blood stasis. Treatment of mice with a series of concentrations of paeonol induced apoptosis of the HepA-xenograft, and meanwhile caused elevation of blood IL-2 and TNF- $\alpha$ in the tumor-bearing mice [38].

Summarizing data from more than 80 independent research papers, it appears that herbal medicines predominately affect apo- ptotic signaling molecules, including increasing the ratio of Bax/ Bcl-2, and upregulating caspases-3, $-8,-9$ and p53/p21 signals. An intriguing observation is that the elevation of PARP-1 cleavage seems to be a common event in cancer cell lines upon the treatment of TCM drugs. It is known that the inhibition of PARP-1 can potentiate both chemo- and radiotherapies for cancer and therefore the search for inhibitor(s) against PARP-1 has been an active area for the development of anticancer drugs [39]. Conversely, excessive expression of PARP-1 can cause translocation of the mitochondrial apoptosis-inducing factor (AIF) to the nuclei, and cause PARP-1-dependent cell death.

There are few herbs that do not cause apoptosis; instead, these herbs (listed in $\odot$ Table 1) generally induce antiangiogenesis, or induce differentiation and change the transforming phenotypes of the tumor cells as listed in O Table 1. According to the TCM classification of medicinal herbs for cancer treatment, we have classified the herb plants listed in $\bullet$ Table 1 into six major classes and show them in Table 2 . To sum up the above actions of the TCM-derived herbal compounds and herbal extracts, we have mapped the found molecular targets from $\bullet$ Table 1 to the known cellular signaling network shown in $\bullet$ Fig. 2 . This schematic diagram shows that MAPK/JNK/p38, JAK/STAT, PI3K/AKTS and NFkappaB are the common signaling pathways affected in responding to the various treatments of TCM. The drawing also illustrates that the caspase family members and the mitochondria-mediated apoptotic molecules might play a role in the anticancer effects of the herbal medicines. However, the information collected up to the time of this review can only offer a rough and incomplete picture of the action of TCM herbal medicines. Further systemic studies are needed for a true and comprehensive understanding of the nature of the TCM products in cancer prevention and treatment. 

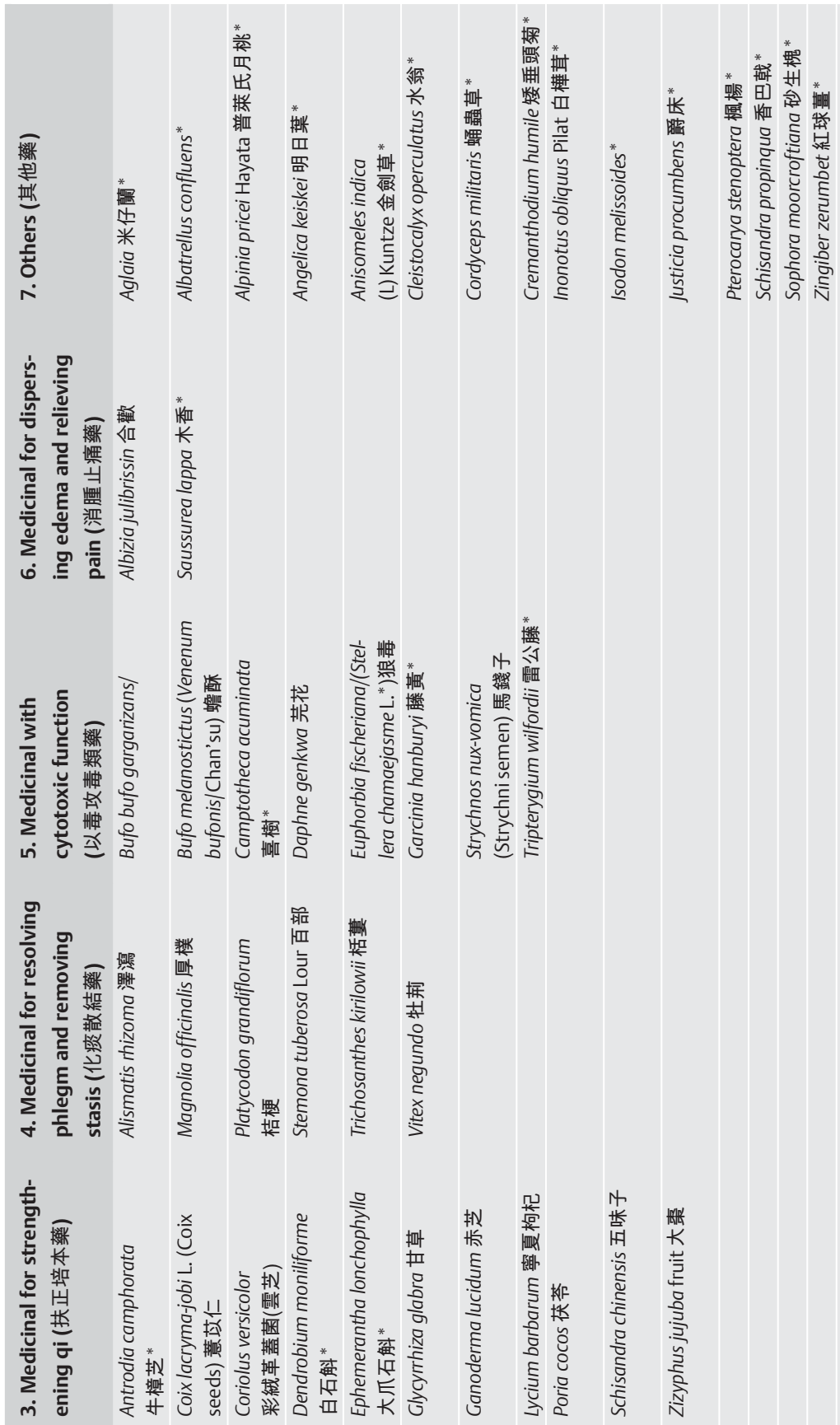

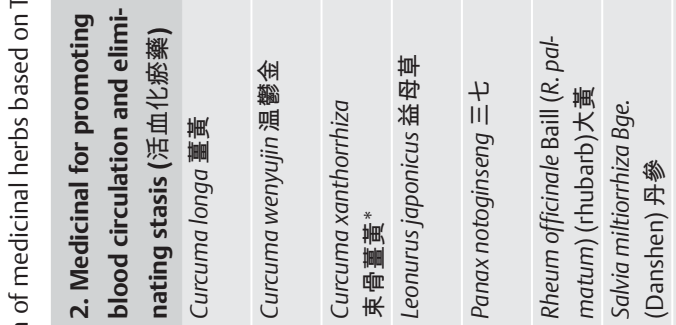

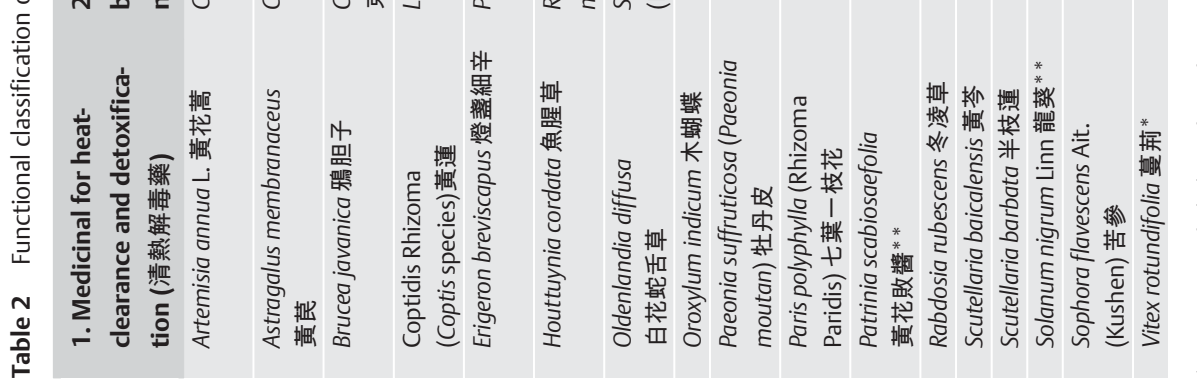




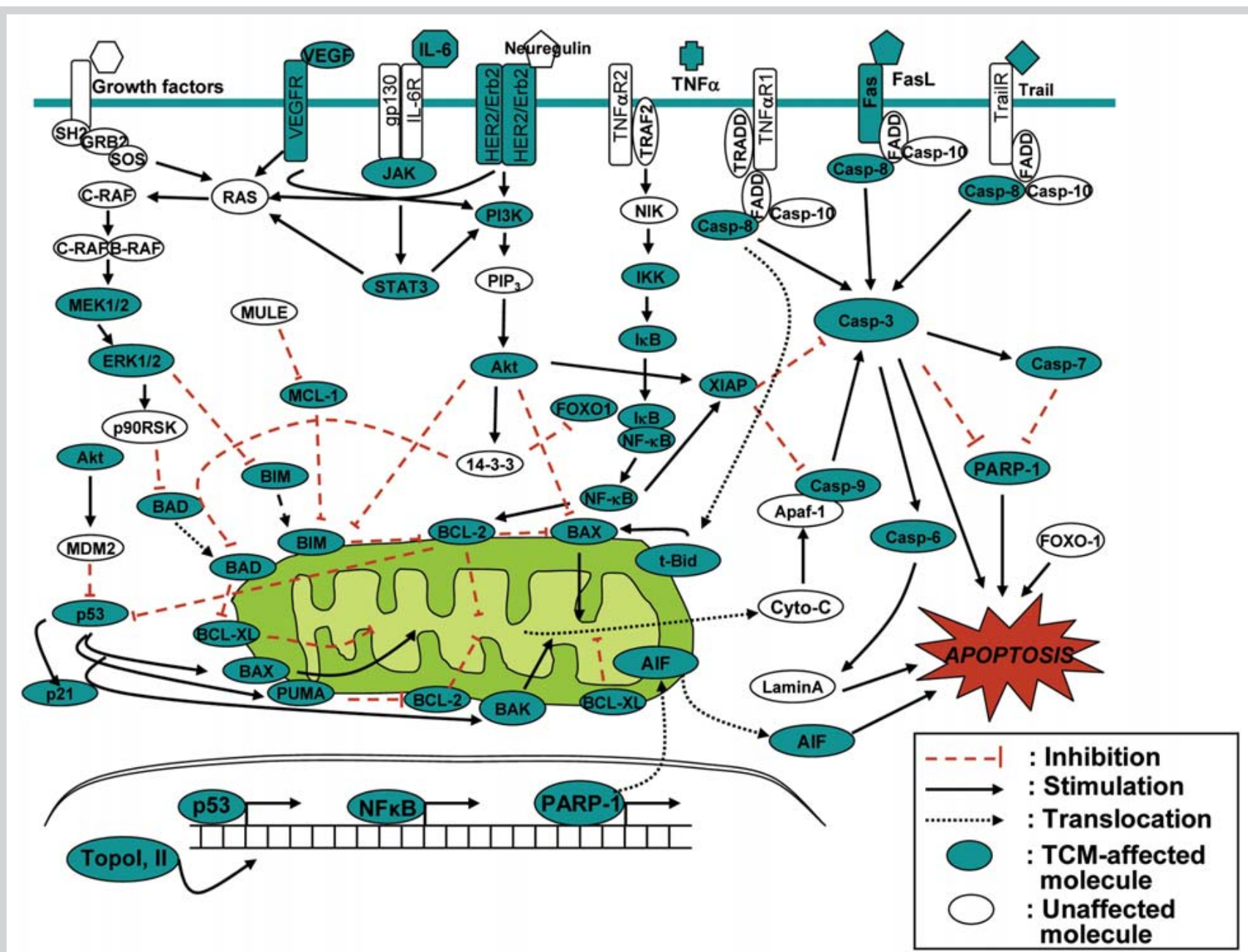

Fig. 2 The altered protein molecules (shown in $\odot$ Table 1) upon treatments of TCM herbal medicines and the associated cellular signaling networks.

\section{Anticancer Effects and Underlying Mechanisms of TCM-Derived Complex Formulas}

There are only a few mechanistic studies on the action of TCM formulas as anticancer agents. One study was on San-ZhongKui-Jian-Tang (SZKJT) [40], a complex formula comprising 17 different herbs, that is used for cancer therapy in China. SZKJT was found to induce the mitochondrial apoptotic pathway by changing Bax/Bcl-2 ratios, cytochrome c release and caspase-9 activation, but did not act on Fas/Fas ligand pathways in two human breast cancer cell lines, MCF-7 and MDA-MB-231. A similar study was carried out by the same laboratory [41] on huang-lian-jiedu-tang (HLJDT) known to possess anti-inflammatory activity. The in vitro study conducted in two human liver cancer cell lines, HepG2 and PLC/PRF/5, found that HLJDT caused cell arrest by upregulating the inactive form of $\mathrm{Cdc} 2$ and $\mathrm{Cdc} 25$, and downregulating the levels of Bcl-2 and Bcl-XL. Furthermore, HLJDT increased the ratio of Bax and Bak/Bcl-2 and Bcl-XL and the associated cell survival pathways, and subsequently triggered the mitochondrial apoptotic pathway. It was the collective actions of the herbs in the formula that were inhibiting the growth of cancer cells tested both in vitro cell lines and in vivo in nude mice. Another study is the study of a classic formula, Guizhi-fuling decoction (GZFLD) [42]. The formulation consists of five herbs: Cinna- momum cassia, Paeonia lactiflora, Paeonia suffruticosa, Poria cocos, and Prunus persica. Accordingly, GZFLD inhibited the growth of HeLa cells by activating the tissue inhibitor of metallopeptidases (TIMPs) and causing the suppression of the activity of the matrix metallopeptidase (MMPs) that play a key role in the degradation of the extracellular matrix and promotion of cell proliferation. In the same study, GZFLD was also shown to inhibit tumor growth and angiogenesis in an in vivo animal model. Another report [43] concerned a classic formula "bojungbangdocktang (BJBDT)" consisting of Astragalus membranaceus Bunge, Atractylodes japonica Koidzumi, Coiz lacryma-jobi Linne var. ma-yuen stapf, Dioscorea batatas Decaisne, Dolichos lablab Linne, Panax ginseng C.A. Mey, Polygonatum sibiricum Delar. ex Pedouté, Poria $\operatorname{cocos}$ (Schw.) Wolf. Two related studies $[44,45]$ found that BJBDT demonstrated antiangiogenesis by blocking VEGF/VEGFR activities in human umbilical vein endothelial cells. Interestingly, BJBDT can prevent cisplatin-induced toxicity and apoptosis in normal MCF-10A, but not in MCF-7 and MDA MB-231 breast cancer cells, suggesting the herbal formula can be applied as a cancer chemopreventive agent [43]. The synergistic effects of herbs in a TCM formula were well illustrated in a recent study, in which a TCM-based formula, Realgar-indigo naturalis (RIF), was applied in the treatment of acute promyelocytic leukemia (APL). The RIF formula has three components, realgar, indigo naturalis, and Sal- 


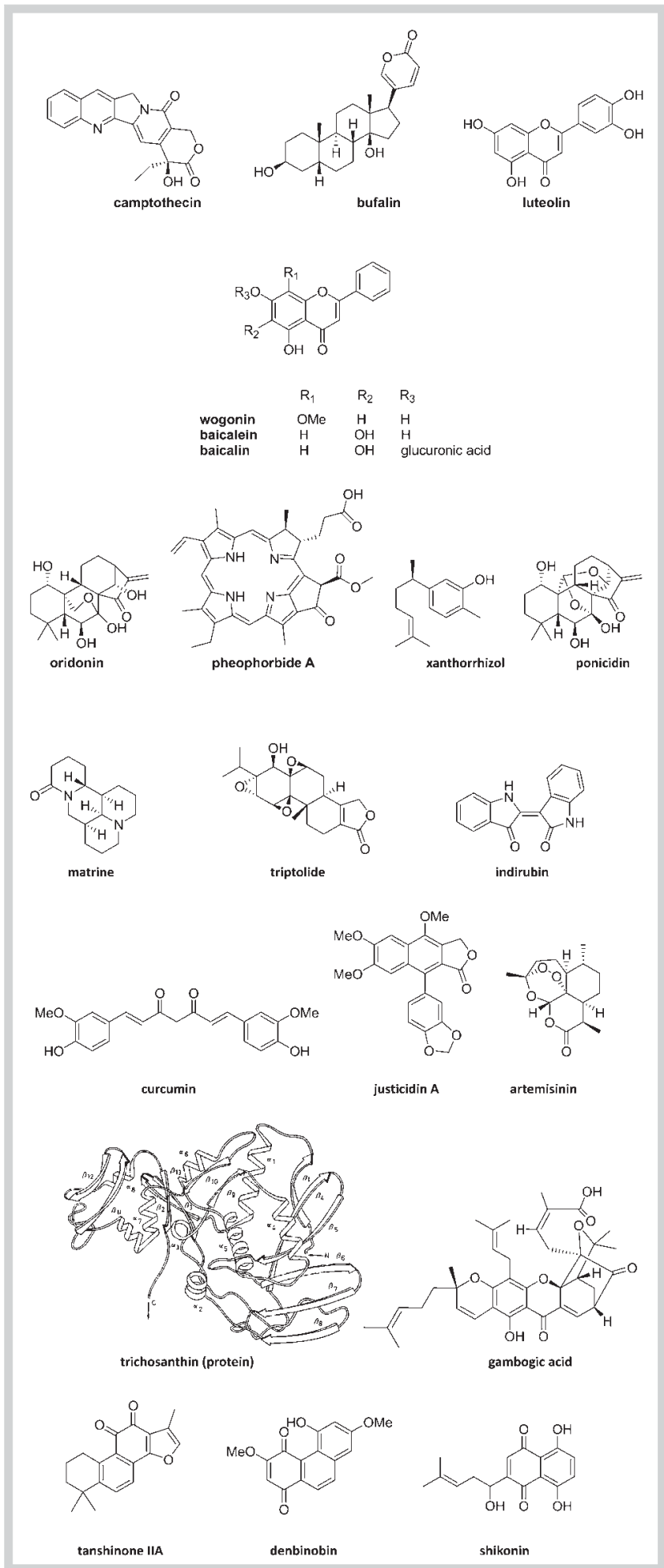

Fig. 3 Chemical structures of the TCM-derived active components shown in $\odot$ Table 1.

via miltiorrhiza of which tetra-arsenic tetrasulfide, indirubin, and tanshinone IIA, respectively, are the major active ingredients [46]. The study demonstrated that tetraarsenic tetrasulfide is the principle component of the formula, while tanshinone IIA and indirubin are the adjuvant ingredients. Together these herbs have shown a synergistic action against APL effective in both in vitro and clinical studies.

\section{Future Prospect of TCM Herbal Medicines in Cancer Research}

The cellular and animal studies have provided strong molecular evidences for the anticancer activities of the TCM herbal medicines, tested as pure compounds or as crude extracts of the single herbs or the complex formulas. However, several important questions remain to be answered. Do TCM-derived herbal medicines possess any special effects other than those often seen with conventional drugs for cancer treatment? There has been little investigation to make a side-by-side comparison. An earlier work was conducted on the anticancer effects of protodioscine (glycosides) from the rhizome of Dioscorea collettii var. hypoglauca, a Chinese herbal remedy for the treatment of cervical carcinoma, carcinoma of urinary bladder and renal tumor for centuries, against a $60 \mathrm{NCI}$ human cancer panel [47], and it was found to be specifically effective for cervical carcinoma, bladder and renal cancer cell lines. Moreover, based on an analysis of the COMPARE computer program with protodioscin as a seed compound, no other compounds in the NCl's anticancer drug screen database have a cytotoxicity pattern (mean graphs) similar to those of protodioscin, indicating that a potential novel mechanism of anticancer action is involved. This may be one of many methods by which the unique properties of TCM can be revealed in a concise manner.

The other question to be addressed in the future is whether the methodologies and the in vitro and in vivo biological models currently employed to investigate the therapeutic nature of traditional Chinese medicines are good enough. In this review, there are 66 herbs that have been used for anticancer studies. We have grouped these herbal plants into seven functional groups based on the traditional usage for cancer treatment ( 0 Table 2). Interestingly only a small subset of herbs is considered toxic, grouped under the category of "medicinal with cytotoxic function", the majority is not. On the other hand, the majority of TCM-derived components shown above are in the same category as the conventional anticancer drugs which induce apoptosis. In a previous study [48], we used a cell system by which the inhibitory effects of non-cytotoxic chemicals were assessed by a focus formation assay upon transfection of ras oncogene to the host cells. Using this system, two well-studied medicinal mushrooms Ganoderma lucidum and Tricholoma lobayense with anticancer potential were examined for their possible adverse effects on cell transformation induced by ras oncogene. The results indicated that both species of mushrooms strongly inhibited ras-induced cell transformation. However, the inhibitory effect of the mushroom extracts was not due to a direct killing of the transformed cells; rather, it seems to have been mediated through the surrounding normal cells. This normal cell-dependent growth inhibitory effect is also observed with oleanolic acid isolated from Oldenlandia diffusa [49]. These examples suggest that, at least some, TCM medicines exert their anticancer effects through mechanism(s) other than apoptosis.

Looking forward, we see three specific issues that will require focused attention: (i) more well-designed clinical trials are required to support the effectiveness and the safety of TCM in the management of cancers; (ii) new parameters based on the unique properties and theory of TCM are needed to assess the clinical ef- 
ficacy of TCM in clinical trials; and (iii) new approaches to research may be needed, given the nature of TCM herbs as being fundamentally different from drugs. There is evidence that the reductionist approach, i.e., searching for one or a few active ingredients in an herb or formula, may not elucidate the efficacy of herbal medicines; a systems biology approach may be more appropriate and productive, in terms of developing effective treatment protocols.

Undoubtedly, the evaluation of the therapeutic effects and the benefits of TCM therapy for cancer patients is a significantly complex, albeit significant, issue. TCM therapy, based on multiple medicinal herbs and an holistic approach to diagnosis as well as treatment, means that a clinical study of TCM treatment is more difficult and complicated than the study of single compound drugs. In addition to the conventional "standards" used for WM clinical trail, there is a need to develop a set of parameters that are suitable to the assessment of TCM therapy. The effects, as well as the toxicity, of individual herbs or, especially, of single compounds derived from the herb cannot completely reflect the benefits and toxicity of the herbal combination. When whole herbs are not studied, improper or biased results and conclusions might be unavoidable [50]. As a goal, to develop TCM into rational cancer therapy, more well-designed intensive clinical evaluations and translational laboratory studies are absolutely needed. And, close collaboration between TCM and conventional Western medicine professions and a combination of TCM with modern multidisciplinary cutting-edge technologies, such as omic methodology on systems biology [51], would provide us with an attractive and effective strategy to achieve this goal.

\section{Acknowledgements}

$\nabla$

The authors thank Dr. William Tai, Ms. Wing Yan Wong, and Dr. Hau Zhou for their technical assistance, and Prof. Zhi Hong Jiang's kind advice on the phytochemistry of the herbs and his contribution to $\odot$ Fig. 3 drawings. Specific thanks go to Dr. Lisa Song's professional assistance in literature search and organization of the references. Thanks also go to Dr. Martha Dahlen's editing of this manuscript. This study was financially supported by Research Grants Council of Hong Kong under HKBU2/07C and HKBU 260307 grants to WLWH.

\section{References}

1 Jemal A, Siegel R, Ward E, Hao Y, Xu J, Thun MJ. Cancer statistics, 2009. CA Cancer J Clin 2009; 59: 225-249

2 Macek $C$. East meets West to balance immunologic yin and yang. JAMA 1984; 251: 433-435, 439

3 Sagar SM, Wong RK. Chinese medicine and biomodulation in cancer patients - part one. Curr Oncol 2008; 15: 42-48

4 Farrell MP, Kummar S. Phase I/IIA randomized study of PHY906, a novel herbal agent, as a modulator of chemotherapy in patients with advanced colorectal cancer. Clin Colorectal Cancer 2003; 2: 253-256

5 Yen Y, So S, Rose M, Saif MW, Chu E, Liu SH, Foo A, Jiang Z, Su T, Cheng YC. Phase I/II study of PHY906/capecitabine in advanced hepatocellular carcinoma. Anticancer Res 2009; 29: 4083-4092

6 Yang YF, Chen ZX, Xu Y, Wu Y, Wu XW, Zhu YW, Li PH, Gao SL. Randomized controlled study on effect of Quxie capsule on the median survival time and qualify of life in patients with advanced colorectal carcinoma. Zhongguo Zhong Xi Yi Jie He Za Zhi 2008; 28: 111-114

7 Tan KY, Liu CB, Chen AH, Ding YJ, Jin HY, Seow-Choen F. The role of traditional Chinese medicine in colorectal cancer treatment. Tech Coloproctol 2008; $12: 1-6$

8 Wu P, Dugoua JJ, Eyawo O, Mills EJ. Traditional Chinese medicines in the treatment of hepatocellular cancers: a systematic review and metaanalysis. J Exp Clin Cancer Res 2009; 28: 112
$9 \mathrm{Xu} Z \mathrm{ZY}$, Jin CJ, Shen DY. Clinical study on treatment of advanced nonsmall-cell lung cancer with Chinese herbal medicine in different stages combined with chemotherapy. Zhongguo Zhong Xi Yi Jie He Za Zhi 2007; 27: 874-878

10 Cheng JH, Liu WS, Li ZM, Wang ZG. A clinical study on global TCM therapy in treating senile advanced non-small cell lung cancer. Chin J Integr Med 2007; 13: 269-274

11 Huang ZF, Wei JS, Li HZ, Tan ZO, Zhang ZJ, Chen C. Effect of Shenqi Fuzheng injection combined with chemotherapy on thirty patients with advanced breast cancer. Zhongguo Zhong Xi Yi Jie He Za Zhi 2008; 28: 152-154

12 Cho WC, Chen HY. Clinical efficacy of traditional Chinese medicine as a concomitant therapy for nasopharyngeal carcinoma: a systematic review and meta-analysis. Cancer Invest 2009; 27: 334-344

13 Liu X, Hua BJ. Effect of traditional Chinese medicine on quality of life and survival period in patients with progressive gastric cancer. Zhongguo Zhong Xi Yi Jie He Za Zhi 2008; 28: 105-107

14 Wei X, Chen Z, Yang X, Wu T. Chinese herbal medicines for esophageal cancer. Cochrane Database Syst Rev 2009; 4: CD004520

15 Liu NL, Chien LY, Tai CJ, Lin KC, Tai CJ. Effectiveness of traditional Chinese medicine for liver protection and chemotherapy completion among cancer patients. eCAM 2009: 1-8

16 Taixiang W, Munro AJ, Guanjian L. Chinese medical herbs for chemotherapy side effects in colorectal cancer patients. Cochrane Database Syst Rev 2005; 1: CD004540

17 Xu L, Lao LX, Ge A, Yu S, Li J, Mansky PJ. Chinese herbal medicine for cancer pain. Integr Cancer Ther 2007; 6: 208-234

18 Wang B, Tian HQ Liang GW. Effect of ganji recipe combined with Fructus Bruceae oil emulsion intervention on quality of life in patients with advanced primary hepatic cancer. Zhongguo Zhong Xi Yi Jie He Za Zhi 2009; 29: 257-260

19 Zhang T. Effect of TCM therapy for removing toxic substance and unblocking meridians on post-radiation quality of life in 55 patients with lung cancer. Zhongguo Zhong Xi Yi Jie He Za Zhi 2008; 28: 154-157

20 Tang W, Hemm I, Bertram B. Recent development of antitumor agents from Chinese herbal medicines; part I. Low molecular compounds. Planta Med 2003; 69: 97-108

21 Tang W, Hemm I, Bertram B. Recent development of antitumor agents from Chinese herbal medicines. Part II. High molecular compounds. Planta Med 2003; 69: 193-201

22 Hsiang YH, Hertzberg R, Hecht S, Liu LF. Camptothecin induces proteinlinked DNA breaks via mammalian DNA topoisomerase I. J Biol Chem 1985; 260: 14873-14878

23 Wall ME. Camptothecin and taxol: discovery to clinic. Med Res Rev 1998; 18: 299-314

24 Oberlies NH, Kroll DJ. Camptothecin and taxol: historic achievements in natural products research. J Nat Prod 2004; 67: 129-135

25 Zhang XW, Qing C, Xu B. Apoptosis induction and cell cycle perturbation in human hepatoma hep G2 cells by 10-hydroxycamptothecin. Anticancer Drugs 1999; 10: 569-576

26 Kaufmann SH, Earnshaw WC. Induction of apoptosis by cancer chemotherapy. Exp Cell Res 2000; 256: 42-49

27 Fulda S, Debatin KM. Targeting inhibitor of apoptosis proteins (IAPs) for diagnosis and treatment of human diseases. Recent Pat Anticancer Drug Discov 2006; 1: 81-89

$28 \mathrm{Ji}$ YB, Gao SY, Ji CF, Zou X. Induction of apoptosis in $\mathrm{HepG}_{2}$ cells by solanine and Bcl-2 protein. J Ethnopharmacol 2008; 115: 194-202

29 Peng CC, Chen KC, Peng RY, Chyau CC, Su CH, Hsieh-Li HM. Antrodia camphorata extract induces replicative senescence in superficial TCC, and inhibits the absolute migration capability in invasive bladder carcinoma cells. J Ethnopharmacol 2007; 109: 93-103

30 Tin MM, Cho CH, Chan K, James AE, Ko JK. Astragalus saponins induce growth inhibition and apoptosis in human colon cancer cells and tumor xenograft. Carcinogenesis 2007; 28: 1347-1355

31 Efferth T. Willmar Schwabe Award 2006: antiplasmodial and antitumor activity of artemisinin - from bench to bedside. Planta Med 2007; 73: 299-309

32 Tang J, Feng Y, Tsao S, Wang N, Curtain R, Wang Y. Berberine and Coptidis rhizoma as novel antineoplastic agents: a review of, traditional use and biomedical investigations. J Ethnopharmacol 2009; 126: 5-17

33 Pandey MK, Sung B, Kunnumakkara AB, Sethi G, Chaturvedi MM, Aggarwal $B B$. Berberine modifies cysteine 179 of IkappaBalpha kinase, suppresses nuclear factor-kappaB-regulated antiapoptotic gene products, and potentiates apoptosis. Cancer Res 2008; 68: 5370-5379 
34 Dai Z, Gao J, Ji Z, Wang X, Ren H, Liu X, Wu W, Kang H, Guan H. Matrine induces apoptosis in gastric carcinoma cells via alteration of Fas/FasL and activation of caspase-3. J Ethnopharmacol 2009; 123: 91-96

35 Kiviharju TM, Lecane PS, Sellers RG, Peehl DM. Antiproliferative and proapoptotic activities of triptolide (PG490), a natural product entering clinical trials, on primary cultures of human prostatic epithelial cells. Clin Cancer Res 2002; 8: 2666-2674

36 Yang M, Huang J, Pan HZ, Jin J. Triptolide overcomes dexamethasone resistance and enhanced PS-341-induced apoptosis via PI3k/Akt/NFkappaB pathways in human multiple myeloma cells. Int J Mol Med 2008; 22: 489-496

37 Baumann S, Fas SC, Giaisi M, Muller WW, Merling A, Gulow K, Edler L, Krammer PH, Li-Weber M. Wogonin preferentially kills malignant lymphocytes and suppresses T-cell tumor growth by inducing PLCgamma1- and $\mathrm{Ca}^{2+}$-dependent apoptosis. Blood 2008; 111: 2354-2363

38 Sun GP, Wang H, Xu SP, Shen YX, Wu Q Chen ZD, Wei W. Anti-tumor effects of paeonol in a HepA-hepatoma bearing mouse model via induction of tumor cell apoptosis and stimulation of IL-2 and TNF-alpha production. Eur J Pharmacol 2008; 584: 246-252

39 Wang Y, Dawson VL, Dawson TM. Poly(ADP-ribose) signals to mitochondrial AIF: a key event in parthanatos. Exp Neurol 2009; 218: 193-202

40 Hsu YL, Yen MH, Kuo PL, Cho CY, Huang YT, Tseng CJ, Lee JP, Lin CC. SanZhong-Kui-Jian-Tang, a traditional Chinese medicine prescription, inhibits the proliferation of human breast cancer cell by blocking cell cycle progression and inducing apoptosis. Biol Pharm Bull 2006; 29: 2388-2394

41 Hsu YL, Kuo PL, Tzeng TF, Sung SC, Yen MH, Lin LT, Lin CC. Huang-lian-jiedu-tang, a traditional Chinese medicine prescription, induces cellcycle arrest and apoptosis in human liver cancer cells in vitro and in vivo. J Gastroenterol Hepatol 2008; 23: e290-e299

42 Yao Z, Shulan Z. Inhibition effect of Guizhi-Fuling-decoction on the invasion of human cervical cancer. J Ethnopharmacol 2008; 120: 25-35

43 Kang S, Jeong S, Kwon H, Yun S, Kim J, Lee H, Lee E, Ahn KS, Kim S. Protective effect of Bojungbangdocktang on cisplatin-induced cytotoxicity and apoptosis in MCF-10A breast endothelial cells. Environ Toxicol Pharmacol 2009; 28: 430-438

44 Lee HJ, Kim KH, Jang YS. Protective effect of ethanol extract of Bojungbangamtang on cisplatin induced toxicity. J Oriental Pathol 2007; 21: $1-5$

45 Jang YS, Lee HJ, Lee HJ, Kim KH, Won SH, Lee JD, Ahn KS, Kim JH, Yu YB, Kim SH. Bojungbangdocktang inhibits vascular endothelial growth factor induced angiogenesis via blocking the VEGF/VEGFR2 signaling pathway in human umbilical vein endothelial cells. Chin Sci Bull 2009; 54: 227-233

46 Wang L, Zhou GB, Liu P, Song JH, Liang Y, Yan XJ, Xu F, Wang BS, Mao JH, Shen ZX, Chen SJ, Chen Z. Dissection of mechanisms of Chinese medicinal formula Realgar-Indigo naturalis as an effective treatment for promyelocytic leukemia. Proc Natl Acad Sci U S A 2008; 105: 4826-4831

$47 \mathrm{Hu} \mathrm{K,} \mathrm{Yao} \mathrm{X.} \mathrm{Protodioscin} \mathrm{(NSC-698} \mathrm{796):} \mathrm{its} \mathrm{spectrum} \mathrm{of} \mathrm{cytotoxicity}$ against sixty human cancer cell lines in an anticancer drug screen panel. Planta Med 2002; 68: 297-301

48 Hsiao WL, Li YQ Lee TL, Li N, You MM, Chang ST. Medicinal mushroom extracts inhibit ras-induced cell transformation and the inhibitory effect requires the presence of normal cells. Carcinogenesis 2004; 25 : $1177-1183$

49 Wu PK, Chi Shing Tai W, Liang ZT, Zhao ZZ, Hsiao WL. Oleanolic acid isolated from Oldenlandia diffusa exhibits a unique growth inhibitory effect against ras-transformed fibroblasts. Life Sci 2009; 85: 113-121

50 Chiu J, Yau T, Epstein RJ. Complications of traditional Chinese/herbal medicines (TCM) - a guide for perplexed oncologists and other cancer caregivers. Support Care Cancer 2009; 17: 231-240

51 Efferth T, Li PC, Konkimalla VS, Kaina B. From traditional Chinese medicine to rational cancer therapy. Trends Mol Med 2007; 13: 353-361

52 O'Leary J, Muggia FM. Camptothecins: a review of their development and schedules of administration. Eur J Cancer 1998; 34: 1500-1508

53 Pastor N, Cortes F. Bufalin influences the repair of X-ray-induced DNA breaks in Chinese hamster cells. DNA Repair (Amst) 2003; 2: 13531360

54 Mizushina Y, Akihisa T, Ukiya M, Murakami C, Kuriyama I, Xu X, Yoshida $H$, Sakaguchi K. A novel DNA topoisomerase inhibitor: dehydroebriconic acid, one of the lanostane-type triterpene acids from Poria cocos. Cancer Sci 2004; 95: 354-360

55 Li CH, Chen PY, Chang UM, Kan LS, Fang WH, Tsai KS, Lin SB. Ganoderic acid $\mathrm{X}$, a lanostanoid triterpene, inhibits topoisomerases and induces apoptosis of cancer cells. Life Sci 2005; 77: 252-265
56 Zhang S, Li X, Zhang F, Yang P, Gao X, Song Q. Preparation of yuanhuacine and relative daphne diterpene esters from Daphne genkwa and structure-activity relationship of potent inhibitory activity against DNA topoisomerase I. Bioorg Med Chem 2006; 14: 3888-3895

57 Meng LH, Ding J. Salvicine, a novel topoisomerase II inhibitor, exerts its potent anticancer activity by ROS generation. Acta Pharmacol Sin 2007; 28: 1460-1465

58 Liang CH, Shiu LY, Chang LC, Sheu HM, Tsai EM, Kuo KW. Solamargine enhances HER2 expression and increases the susceptibility of human lung cancer H661 and H69 cells to trastuzumab and epirubicin. Chem Res Toxicol 2008; 21: 393-399

$59 \mathrm{Li}$-Weber M. New therapeutic aspects of flavones: the anticancer properties of Scutellaria and its main active constituents wogonin, baicalein and baicalin. Cancer Treat Rev 2009; 35: 57-68

60 Lopez-Lazaro M. Distribution and biological activities of the flavonoid luteolin. Mini Rev Med Chem 2009; 9: 31-59

61 Chow LW, Lo CS, Loo WT, Hu XC, Sham JS. Polysaccharide peptide mediates apoptosis by up-regulating p 21 gene and down-regulating cyclin D1 gene. Am J Chin Med 2003; 31: 1-9

62 Yin X, Zhou J, Jie C, Xing D, Zhang Y. Anticancer activity and mechanism of Scutellaria barbata extract on human lung cancer cell line A549. Life Sci 2004; 75: 2233-2244

63 Liu JJ, Huang RW, Lin DJ, Wu XY, Peng J, Pan XL, Song YQ Lin Q Hou M, Wang DN, Chen F, Zhang MH. Oridonin-induced apoptosis in leukemia K562 cells and its mechanism. Neoplasma 2005; 52: 225-230

64 Chan JY, Tang PM, Hon PM, Au SW, Tsui SK, Waye MM, Kong SK, Mak TC, Fung KP. Pheophorbide a, a major antitumor component purified from Scutellaria barbata, induces apoptosis in human hepatocellular carcinoma cells. Planta Med 2006; 72: 28-33

65 Cheah YH, Azimahtol HLP, Abdullah NR. Xanthorrhizol exhibits antiproliferative activity on MCF-7 breast cancer cells via apoptosis induction. Anticancer Res 2006; 26: 4527-4534

66 Chiu LC, Ho TS, Wong EY, Ooi VE. Ethyl acetate extract of Patrinia scabiosaefolia downregulates anti-apoptotic $\mathrm{Bcl}-2 / \mathrm{Bcl}-\mathrm{X}(\mathrm{L})$ expression, and induces apoptosis in human breast carcinoma MCF-7 cells independent of caspase-9 activation. J Ethnopharmacol 2006; 105: 263-268

67 Huang YT, Huang DM, Chueh SC, Teng CM, Guh JH. Alisol B acetate, a triterpene from Alismatis rhizoma, induces Bax nuclear translocation and apoptosis in human hormone-resistant prostate cancer PC-3 cells. Cancer Lett 2006; 231: 270-278

68 Liu J, Huang R, Lin D, Peng J, Zhang M, Pan X, Hou M, Wu X, Lin Q Chen F. Ponicidin, an ent-kaurane diterpenoid derived from a constituent of the herbal supplement PC-SPES, Rabdosia rubescens, induces apoptosis by activation of caspase- 3 and mitochondrial events in lung cancer cells in vitro. Cancer Invest 2006; 24: 136-148

69 Liu XS, Jiang J. Molecular mechanism of matrine-induced apoptosis in leukemia K562 cells. Am J Chin Med 2006; 34: 1095-1103

70 Panichakul T, Intachote P, Wongkajorsilp A, Sripa B, Sirisinha S. Triptolide sensitizes resistant cholangiocarcinoma cells to TRAIL-induced apoptosis. Anticancer Res 2006; 26: 259-265

71 Perabo FGE, Frossler C, Landwehrs G, Schmidt DH, Von Rucker A, Wirger $A$, Muller SC. Indirubin-3'-monoxime, a CDK inhibitor induces growth inhibition and apoptosis-independent up-regulation of survivin in transitional cell cancer. Anticancer Res 2006; 26: 2129-2135

72 Rajasingh J, Raikwar HP, Muthian G, Johnson C, Bright JJ. Curcumin induces growth-arrest and apoptosis in association with the inhibition of constitutively active JAK-STAT pathway in T cell leukemia. Biochem Biophys Res Commun 2006; 340: 359-368

73 Su CL, Huang LL, Huang LM, Lee JC, Lin CN, Won SJ. Caspase-8 acts as a key upstream executor of mitochondria during justicidin A-induced apoptosis in human hepatoma cells. FEBS Lett 2006; 580: 3185-3191

74 Tan TW, Tsai HR, Lu HF, Lin HL, Tsou MF, Lin YT, Tsai HY, Chen YF, Chung JG. Curcumin-induced cell cycle arrest and apoptosis in human acute promyelocytic leukemia HL-60 cells via MMP changes and caspase-3 activation. Anticancer Res 2006; 26: 4361-4371

75 Tang W, Liu JW, Zhao WM, Wei DZ, Zhong JJ. Ganoderic acid T from Ganoderma lucidum mycelia induces mitochondria mediated apoptosis in lung cancer cells. Life Sci 2006; 80: 205-211

76 Wang X, Matta R, Shen G, Nelin LD, Pei D, Liu Y. Mechanism of triptolideinduced apoptosis: Effect on caspase activation and Bid cleavage and essentiality of the hydroxyl group of triptolide. J Mol Med 2006; 84: 405-415

77 Won HJ, Han CH, Kim YH, Kwon HJ, Kim BW, Choi JS, Kim K. Induction of apoptosis in human acute leukemia Jurkat T cells by Albizzia julibrissin 
extract is mediated via mitochondria-dependent caspase-3 activation. J Ethnopharmacol 2006; 106: 383-389

78 Yadav SK, Lee SC. Evidence for Oldenlandia diffusa-evoked cancer cell apoptosis through superoxide burst and caspase activation. J Chin Integr Med 2006; 4: 485-489

79 Yang HL, Chen CS, Chang WH, Lu FJ, Lai YC, Chen CC, Hseu TH, Kuo CT, Hseu YC. Growth inhibition and induction of apoptosis in MCF-7 breast cancer cells by Antrodia camphorata. Cancer Lett 2006; 231: 215-227

80 Jin S, Pang RP, Shen JN, Huang G, Wang J, Zhou JG. Grifolin induces apoptosis via inhibition of PI3K/AKT signalling pathway in human osteosarcoma cells. Apoptosis 2007; 12: 1317-1326

81 Huang X, Kojima-Yuasa A, Norikura T, Kennedy DO, Hasuma T, MatsuiYuasa I. Mechanism of the anti-cancer activity of Zizyphus jujuba in HepG2 cells. Am J Chin Med 2007; 35: 517-532

82 Ko JKS, Leung WC, Ho WK, Chiu P. Herbal diterpenoids induce growth arrest and apoptosis in colon cancer cells with increased expression of the nonsteroidal anti-inflammatory drug-activated gene. Eur J Pharmacol 2007; 559: 1-13

83 Kuo PL, Hsu YL, Sung SC, Ni WC, Lin TC, Lin CC. Induction of apoptosis in human breast adenocarcinoma MCF-7 cells by pterocarnin A from the bark of Pterocarya stenoptera via the Fas-mediated pathway. Anticancer Drugs 2007; 18: 555-562

84 Li H, Wang LJ, Qiu GF, Yu JQ Liang SC, Hu XM. Apoptosis of Hela cells induced by extract from Cremanthodium humile. Food Chem Toxicol 2007; 45: 2040-2046

$85 \mathrm{LiJ}$, Xia X, Ke Y, Nie H, Smith MA, Zhu X. Trichosanthin induced apoptosis in HL-60 cells via mitochondrial and endoplasmic reticulum stress signaling pathways. Biochim Biophys Acta Gen Subj 2007; 1770: 11691180

86 Li Z, Sturm S, Stuppner H, Schraml E, Moser VA, Siegl V, Pfragner R. The dichloromethane fraction of Stemona tuberosa Lour inhibits tumor cell growth and induces apoptosis of human medullary thyroid carcinoma cells. Biologics 2007; 1: 455-463

87 Lin J, Chen LY, Lin ZX, Zhao ML. The effect of triptolide on apoptosis of glioblastoma multiforme (GBM) cells. J Int Med Res 2007; 35: 637-643

$88 \mathrm{Mu}$ D, Chen $W, Y u$ B, Zhang C, Zhang Y, Qi H. Calcium and survivin are involved in the induction of apoptosis by dihydroartemisinin in human lung cancer SPC-A-1 cells. Methods Find Exp Clin Pharmacol 2007; 29: $33-38$

89 Nishimura R, Tabata K, Arakawa M, Ito Y, Kimura Y, Akihisa T, Nagai H, Sakuma A, Kohno H, Suzuki T. Isobavachalcone, a chalcone constituent of Angelica keiskei, induces apoptosis in neuroblastoma. Biol Pharm Bull 2007; 30: 1878-1883

90 Roy MK, Nakahara K, Na Thalang V, Trakoontivakorn G, Takenaka M, Isobe $S$, Tsushida $T$. Baicalein, a flavonoid extracted from a methanolic extract of Oroxylum indicum inhibits proliferation of a cancer cell line in vitro via induction of apoptosis. Pharmazie 2007; 62: 149-153

91 Tao Z, Zhou Y, Lu J, Duan W, Qin Y, He X, Lin L, Ding J. Caspase-8 preferentially senses the apoptosis-inducing action of NG-18, a gambogic acid derivative, in human leukemia HL-60 cells. Cancer Biol Ther 2007; 6: 691-696

92 Tian Z, Xu L, Zhou L, Yang M, Chen S, Xiao P, Wu E. Cytotoxic activity of schisandrolic and isoschisandrolic acids involves induction of apoptosis. Chemotherapy 2007; 53: 257-262

93 Wang J, Wang X, Jiang S, Yuan S, Lin P, Zhang J, Lu Y, Wang Q Xiong Z, Wu $Y$, Ren J, Yang $H$. Growth inhibition and induction of apoptosis and differentiation of tanshinone IIA in human glioma cells. J Neurooncol 2007; 82: 11-21

94 Xiao Y, Yang F, Li S, Gao J, Hu G, Lao S, Conceicao EL, Fung K, Wang Y, Lee $S M$. Furanodiene induces $\mathrm{G}_{2} / \mathrm{M}$ cell cycle arrest and apoptosis through MAPK signaling and mitochondria-caspase pathway in human hepatocellular carcinoma cells. Cancer Biol Ther 2007; 6: 1044-1050

95 Yang $L$, Wu S, Zhang Q Liu F, Wu P. 23,24-Dihydrocucurbitacin B induces $\mathrm{G}_{2} / \mathrm{M}$ cell-cycle arrest and mitochondria-dependent apoptosis in human breast cancer cells (Bcap37). Cancer Lett 2007; 256: 267-278

96 Yu ZY, Liang YG, Xiao H, Shan YJ, Dong B, Huang R, Fu YL, Zhao ZH, Liu ZY, Zhao QS, Wang SQ Chen JP, Mao BZ, Cong YW. Melissoidesin G, a diterpenoid purified from Isodon melissoides, induces leukemic-cell apoptosis through induction of redox imbalance and exhibits synergy with other anticancer agents. Int J Cancer 2007; 121: 2084-2094

97 Zhu JY, Lavrik IN, Mahlknecht U, Giaisi M, Proksch P, Krammer PH, LiWeber $M$. The traditional Chinese herbal compound rocaglamide preferentially induces apoptosis in leukemia cells by modulation of mitogen-activated protein kinase activities. Int J Cancer 2007; 121: 18391846
98 Carter BZ, Mak DH, Schober WD, Dietrich MF, Pinilla C, Vassilev LT, Reed $J C$, Andreeff $M$. Triptolide sensitizes AML cells to TRAIL-induced apoptosis via decrease of XIAP and p53-mediated increase of DR5. Blood 2008; 111: 3742-3750

99 Chen TH, Pan SL, Guh JH, Chen CC, Huang YT, Pai HC, Teng CM. Denbinobin induces apoptosis by apoptosis-inducing factor releasing and DNA damage in human colorectal cancer HCT-116 cells. Naunyn Schmiedebergs Arch Pharmacol 2008; 378: 447-457

100 Eom KS, Hong JM, Youn MJ, So HS, Park R, Kim JM, Kim TY. Berberine induces G1 arrest and apoptosis in human glioblastoma T98G cells through mitochondrial/caspases pathway. Biol Pharm Bull 2008; 31: 558-562

101 Hahm ER, Arlotti JA, Marynowski SW, Singh SV. Honokiol, a constituent of oriental medicinal herb magnolia officinalis, inhibits growth of PC-3 xenografts in vivo in association with apoptosis induction. Clin Cancer Res 2008; 14: 1248-1257

102 Ho CM, Huang CC, Huang CJ, Cheng JS, Chen IS, Tsai JY, Jiann BP, Tseng PL, Kuo SJ, Jan CR. Effects of Antrodia camphorata on viability, apoptosis, and $\left[\mathrm{Ca}^{2+}\right]$ i in PC3 human prostate cancer cells. Chin J Physiol 2008; 51: 78-84

103 Jin CY, Kim GY, Choi YH. Induction of apoptosis by aqueous extract of Cordyceps militaris through activation of caspases and inactivation of Akt in human breast cancer MDA-MB-231 cells. J Microbiol Biotechnol 2008; 18: 1997-2003

104 Kim DC, Ramachandran S, Baek SH, Kwon SH, Kwon KY, Cha SD, Bae I, $\mathrm{Cho} \mathrm{CH}$. Induction of growth inhibition and apoptosis in human uterine leiomyoma cells by isoliquiritigenin. Reprod Sci 2008; 15: 552558

105 Kim EJ, Lim SS, Park SY, Shin HK, Kim JS, Park JH. Apoptosis of DU145 human prostate cancer cells induced by dehydrocostus lactone isolated from the root of Saussurea lappa. Food Chem Toxicol 2008; 46: 3651-3658

106 Kuo CT, Hsu MJ, Chen BC, Chen CC, Teng CM, Pan SL, Lin CH. Denbinobin induces apoptosis in human lung adenocarcinoma cells via Akt inactivation, Bad activation, and mitochondrial dysfunction. Toxicol Lett 2008; 177 : 48-58

107 Lau ST, Lin ZX, Zhao M, Leung PS. Brucea javanica fruit induces cytotoxicity and apoptosis in pancreatic adenocarcinoma cell lines. Phytother Res 2008; 22: 477-486

108 Lee SM, Kwon II, Choi YH, Eom HS, Chi GY. Induction of G2/M arrest and apoptosis by water extract of Strychni Semen in human gastric carcinoma AGS cells. Phytother Res 2008; 22: 752-758

109 Lin S, Fujii M, Hou D. Molecular mechanism of apoptosis induced by schizandrae-derived lignans in human leukemia HL-60 cells. Food Chem Toxicol 2008; 46: 590-597

110 Liu WK, Cheung FW, Liu BP, Li C, Ye W, Che CT. Involvement of p21 and FasL in induction of cell cycle arrest and apoptosis by neochamaejasmin A in human prostate LNCaP cancer cells. J Nat Prod 2008; 71: $842-846$

111 Min R, Tong J, Wenjun Y, Wenhu D, Xiaojian Z, Jiacai H, Jian Z, Wantao C, Chenping $Z$. Growth inhibition and induction of apoptosis in human oral squamous cell carcinoma Tca-8113 cell lines by shikonin was partly through the inactivation of NF-kappaB pathway. Phytother Res 2008; 22: 407-415

112 Mu D, Zhang W, Chu D, Liu T, Xie Y, Fu E, Jin F. The role of calcium, P38 MAPK in dihydroartemisinin-induced apoptosis of lung cancer PC-14 cells. Cancer Chemother Pharmacol 2008; 61: 639-645

113 Sagawa M, Nakazato T, Uchida H, Ikeda Y, Kizaki M. Cantharidin induces apoptosis of human multiple myeloma cells via inhibition of the JAK/STAT pathway. Cancer Sci 2008; 99: 1820-1826

114 Yan SS, Li Y, Wang Y, Shen SS, Gu Y, Wang HB, Qin GW, Yu Q. 17-Acetoxyjolkinolide B irreversibly inhibits IkappaB kinase and induces apoptosis of tumor cells. Mol Cancer Ther 2008; 7: 1523-1532

115 Yang HL, Chen SC, Chen CS, Wang SY, Hseu YC. Alpinia pricei rhizome extracts induce apoptosis of human carcinoma KB cells via a mitochondria-dependent apoptotic pathway. Food Chem Toxicol 2008; 46: 3318-3324

116 Yang M, Huang J, Pan H, Jin J. Triptolide overcomes dexamethasone resistance and enhanced PS-341-induced apoptosis via PI3k/Akt/NFkappaB pathways in human multiple myeloma cells. Int J Mol Med 2008; 22: 489-496

117 Abdel Wahab SI, Abdul AB, Alzubairi AS, Mohamed Elhassan M, Mohan S. In vitro ultramorphological assessment of apoptosis induced by Zerumbone on (HeLa). J Biomed Biotechnol 2009; 2009: 769568 
118 Chan JY, Tan BKH, Lee SC. Scutellarin sensitizes drug-evoked colon cancer cell apoptosis through enhanced caspase-6 activation. Anticancer Res 2009; 29: 3043-3047

119 Dai ZJ, Gao J, Ji ZZ, Wang XJ, Ren HT, Liu XX, Wu WY, Kang HF, Guan HT. Matrine induces apoptosis in gastric carcinoma cells via alteration of Fas/FasL and activation of caspase-3. J Ethnopharmacol 2009; 123: 91-96

120 Hou YY, Wu ML, Hwang YC, Chang FR, Wu YC, Wu CC. The natural diterpenoid ovatodiolide induces cell cycle arrest and apoptosis in human oral squamous cell carcinoma Ca9-22 cells. Life Sci 2009; 85: 26-32

121 Lai WW, Yang JS, Lai KC, Kuo CL, Hsu CK, Wang CK, Chang CY, Lin JJ, Tang $N Y$, Chen PY, Huang WW, Chung JG. Rhein induced apoptosis through the endoplasmic reticulum stress, caspase- and mitochondria-dependent pathways in SCC-4 human tongue squamous cancer cells. In Vivo 2009; 23: 309-316

122 Lee DH, Rhee JG, Lee YJ. Reactive oxygen species up-regulate p53 and Puma; a possible mechanism for apoptosis during combined treatment with TRAIL and wogonin. Br J Pharmacol 2009; 157: 1189-1202

123 Li DD, Wu XQ Tang J, Wei XY, Zhu XF. ON-III inhibits erbB-2 tyrosine kinase receptor signal pathway and triggers apoptosis through induction of Bim in breast cancer cells. Cancer Biol Ther 2009; 8: 739-743

124 Liu W, Mu R, Nie FF, Yang Y, Wang J, Dai QS, Lu N, Qi Q Rong JJ, Hu R, Wang XT, You QD, Guo QL. MAC-related mitochondrial pathway in oroxylin-A-induced apoptosis in human hepatocellular carcinoma HepG2 cells. Cancer Lett 2009; 284: 198-207

125 Lu YY, Chen TS, Qu JL, Pan WL, Sun L, Wei XB. Dihydroartemisinin (DHA) induces caspase-3-dependent apoptosis in human lung adenocarcinoma ASTC-a-1 cells. J Biomed Sci 2009; 16: 16

126 Luo Q Li Z, Yan J, Zhu F, Xu RJ, Cai YZ. Lycium barbarum polysaccharides induce apoptosis in human prostate cancer cells and inhibits prostate cancer growth in a xenograft mouse model of human prostate cancer. J Med Food 2009; 12: 695-703

127 Park SC, Yoo HS, Park C, Cho CK, Kim G, Kim W, Lee Y, Choi YH. Induction of apoptosis in human lung carcinoma cells by the water extract of Panax notoginseng is associated with the activation of caspase-3 through downregulation of Akt. Int J Oncol 2009; 35: 121-127

128 Sanchez-Duffhues G, Calzado MA, de Vinuesa AG, Appendino G, Fiebich BL, Loock U, Lefarth-Risse A, Krohn K, Munoz E. Denbinobin inhibits nuclear factor-kappaB and induces apoptosis via reactive oxygen species generation in human leukemic cells. Biochem Pharmacol 2009; 77: 1401-1409

129 Shen JK, Du HP, Yang M, Wang YG, Jin J. Casticin induces leukemic cell death through apoptosis and mitotic catastrophe. Ann Hematol 2009; 88: 743-752

130 Shin DY, Kim GY, Li W, Choi BT, Kim ND, Kang HS, Choi YH. Implication of intracellular ROS formation, caspase-3 activation and Egr- 1 induction in platycodon D-induced apoptosis of U937 human leukemia cells. Biomed Pharmacother 2009; 63: 86-94

131 Tang YJ, Yang JS, Lin CF, Shyu WC, Tsuzuki M, Lu CC, Chen YF, Lai KC. Houttuynia cordata Thunb extract induces apoptosis through mitochondrial-dependent pathway in HT-29 human colon adenocarcinoma cells. Oncol Rep 2009; 22: 1051-1056

132 Tsang CM, Lau EPW, Di K, Cheung PY, Hau PM, Ching YP, Wong YC, Cheung ALM, Wan TSK, Tong Y, Tsao SW, Feng Y. Berberine inhibits Rho GTPases and cell migration at low doses but induces G2 arrest and apoptosis at high doses in human cancer cells. Int J Mol Med 2009; 24: 131-138

133 Wang Y, Ma X, Yan S, Shen S, Zhu H, Gu Y, Wang H, Qin G, Yu Q. 17-Hydroxy-jolkinolide $B$ inhibits signal transducers and activators of transcription 3 signaling by covalently cross-linking Janus kinases and induces apoptosis of human cancer cells. Cancer Res 2009; 69: 73027310

134 Wong BYY, Nguyen DL, Lin T, Wong HHL, Cavalcante A, Greenberg NM, Hausted RP, Zheng J. Chinese medicinal herb Scutellaria barbata modulates apoptosis and cell survival in murine and human prostate cancer cells and tumor development in TRAMP mice. Eur J Cancer Prev 2009; 18: 331-341

135 Xiao X, Bai P, Bui Nguyen TM, Xiao J, Liu S, Yang G, Hu L, Chen X, Zhang X, Liu J, Wang $H$. The antitumoral effect of Paris Saponin I associated with the induction of apoptosis through the mitochondrial pathway. Mol Cancer Ther 2009; 8: 1179-1188

136 Xie H, Qin YX, Zhou YL, Tong LJ, Lin LP, Geng MY, Duan WH, Ding J. GA3, a new gambogic acid derivative, exhibits potent antitumor activities in vitro via apoptosis-involved mechanisms. Acta Pharmacol Sin 2009; 30: $346-354$

137 Xu X, Liu Y, Wang L, He J, Zhang H, Chen X, Li Y, Yang J, Tao J. Gambogic acid induces apoptosis by regulating the expression of Bax and Bcl-2 and enhancing caspase-3 activity in human malignant melanoma A375 cells. Int J Dermatol 2009; 48: 186-192

138 Yun HR, Yoo HS, Shin DY, Hong SH, Kim JH, Cho CK, Choi YH. Apoptosis induction of human lung carcinoma cells by Chan Su (Venenum bufonis) through activation of caspases. JAMS J Acupunct Meridian Stud 2009; 2: 210-217

139 Zhou Y, Yiliang EL, Cao J, Zeng G, Shen C, Li Y, Zhou M, Chen Y, Pu W, Potters L, Shi YE. Vitexins, nature-derived lignan compounds, induce apoptosis and suppress tumor growth. Clin Cancer Res 2009; 15 : 5161-5169

140 Jing Y, Watabe M, Hashimoto S, Nakajo S, Nakaya K. Cell cycle arrest and protein kinase modulating effect of bufalin on human leukemia ML1 cells. Anticancer Res 1994; 14: 1193-1198

141 Woo JH, Li D, Wilsbach K, Orita H, Coulter J, Tully E, Kwon TK, Xu S, Gabrielson $E$. Coix seed extract, a commonly used treatment for cancer in China, inhibits NFkappaB and protein kinase C signaling. Cancer Biol Ther 2007; 6: 2005-2011

142 Que HF, Chen HF, Gao SP, Lu DM, Tang HJ, Jia XH, Xu JN. Effect on runing II on the growth of metastasis of transplanted tumor in mammary cancer-bearing mice and its mechanism. J Tradit Chin Med 2008; 28 293-298

$143 \mathrm{Lu}$ Q Zhang P, Zhang X, Chen J. Experimental study of the anti-cancer mechanism of tanshinone IIA against human breast cancer. Int J Mol Med 2009; 24: 773-780

144 Tao J, Zhang P, Liu G, Yan H, Bu X, Ma Z, Wang N, Jia W. Cytotoxicity of Chinese motherwort (YiMuCao) aqueous ethanol extract is, non-apoptotic and estrogen receptor independent on human breast cancer cells. J Ethnopharmacol 2009; 122: 234-239

145 Yo YT, Shieh GS, Hsu KF, Wu CL, Shiau AL. Licorice and licochalcone-A induce autophagy in LNCaP prostate cancer cells by suppression of Bcl-2 expression and the mTOR pathway. J Agric Food Chem 2009; 57: 8266-8273 